\title{
Murciélagos del Ecuador: un análisis de sus patrones de riqueza, distribución y aspectos de conservación
}

Santiago F. Burneo ${ }^{1,2^{*}}$ y Diego G. Tirira ${ }^{1,2,3}$

Introduction: The knowledge of the distribution of bat species in Ecuador has changed significantly in recent years, from new records to taxonomic revisions that have shaped distribution maps to a broader biogeographic understanding. A review of the richness patterns and potential distribution of bats in Ecuador is presented, based on the analysis of records published or stored in scientific collections using Ecological Niche Modeling (ENM) tools. Although maps resulting from ENM are limited both because of the lack of equilibrium and limitations on the representativeness of samples, they constitute a better depiction of distribution than minimal convex polygons or altitudinal ranges. According to the Ecuadorian Red List, 19 species of bats are currently threatened, mainly because of habitat conversion as a consequence of recent colonization, so a better understanding of distribution and spatial richness will result in better proposals for research-priority and conservation-priority areas.

Methodology: The analysis was based on 21,455 records, corresponding to 162 species. This information was reviewed and validated using Geographic Information Systems. A maximum entropy algorithm implemented in Maxent was used to evaluate and generate potential distribution models of the species. Those species with insufficient data to generate a model or for which the evaluation was unsatisfactory were eliminated from the analysis. The remaining species models were used to create a composite map representing the richness of bat species for Ecuador, which in turn was used to assess the conservation status of bat diversity in the country.

Results: Following review and validation of the data, 10,916 records were used to determine the potential distribution of 81 species of bats, based on ENM. A map of potential bat species richness was obtained for the country with the overlap of the models, representing areas that due to climatic conditions, allow a higher or lower species richness of bats living in sympatry. We determined that the central and north-eastern foothills of the Andes are the most suitable areas enclosing the highest richness of bats in Ecuador. Researchpriority and conservation-priority areas were identified.

Discussion and conclusions: Information on protected areas of Ecuador was overlaid on top of the potential richness map, showing that only $5.6 \%$ of the area with the greatest potential bat richness is protected. Accordingly, we determine the existing information gaps and identify priority areas for research and conservation of bats in Ecuador. Three research-priority areas were defined: (1) the Southeastern tropics, between Pastaza and Morona Santiago provinces; (2) the Northern Andes towards the cordillera's western slopes; and (3) areas of the Western dry tropical forests, between the provinces of Guayas and Manabí. Three conservation priority areas were defined:(1) northwest from the province of Pichincha with patches of forest sufficiently large and connected to be protected; (2) most of the Pastaza province, south of the Yasuní National Park; and (3) from the central region of the Morona Santiago province to the northern half of the province of Zamora, towards the Peruvian border to the east.

Key words: Amazonia, deforestation, modeling, priority areas, protected areas, scientific collections.

\footnotetext{
${ }^{1}$ Museo de Zoología, Pontificia Universidad Católica del Ecuador. Av. 12 de Octubre 1076 y Roca, 170517, Quito, Ecuador. (593-2) 255-6560. E-mail: sburneo@puce.edu.ec

${ }^{2}$ Programa para la Conservación de los Mamíferos del Ecuador

${ }^{3}$ Fundación Mamíferos y Conservación. Víctor Balseca 100 y General Gribaldo Miño, 170809, Conocoto, Quito, Ecuador. (593-2) 209-9127. E-mail: diego_tirira@yahoo.com

"Corresponding author
} 
Se presenta una revisión de los patrones de riqueza y de distribución potencial de murciélagos en el Ecuador, basado en el análisis de 21,455 registros publicados o almacenados en colecciones científicas, correspondientes a 162 especies. Luego de la revisión y validación de los datos, se procesaron 10,916 registros que sirvieron para conocer la distribución potencial de 81 especies de quirópteros, basada en el modelamiento predictivo de nicho. Mediante la superposición de los 81 modelos se obtuvo un mapa de riqueza potencial de murciélagos que representa aquellas zonas en el país que, por sus condiciones climáticas, permitirían una mayor o menor riqueza de especies de murciélagos en simpatría. Con esta información, se determinó que las estribaciones centro y nororientales de los Andes, entre los 250 y 1,800 m de altitud, son áreas de mayor diversidad de murciélagos por su idoneidad de hábitat. La información de riqueza potencial fue superpuesta con el mapa de áreas protegidas de Ecuador, lo cual evidenció que apenas un 5.6\% del área con mayor riqueza potencial de quirópteros se encuentra protegida. También se discuten aspectos de conservación en relación con las amenazas que en la actualidad enfrenta la vida silvestre en Ecuador y que en mayor medida afectan al Orden Chiroptera. Es así que se determinaron los vacíos de información existentes y se definieron áreas prioritarias de investigación y conservación de murciélagos en el Ecuador.

Palabras clave: Amazonía, áreas prioritarias, áreas protegidas, colecciones científicas, deforestación, modelamientos.

Introducción

El conocimiento de los patrones de distribución de las especies de murciélagos presentes en Ecuador ha cambiado sustancialmente en las últimas décadas. Obras como las revisiones de Albuja (1982, 1999), Tirira (1999, 2007) y Gardner (2008), han contribuido de manera sustancial a un mejor entendimiento de la distribución de muchas especies; aportes que se han visto fortalecidos con numerosas revisiones y estudios que han aparecido en los últimos años (entre ellos Reid et al. 2000; Albuja y Mena-V. 2004; Lee et al. 2006a, b; Lee et al. 2008; Tirira 2008; Lee et al. 2010; Carrera et al. 2010). Sin embargo, todos los esfuerzos previos por explicar la distribución de las especies de murciélagos en el Ecuador se han basado únicamente en registros de colecciones e intervalos altitudinales; sin considerarse patrones ecológicos y ecosistémicos, formaciones vegetales o características bioclimáticas, que ayuden a un mejor entendimiento de la distribución potencial de los quirópteros en el país.

Para analizar patrones de distribución y de riqueza de un grupo de seres vivos es necesario partir del concepto de área de distribución de una especie, la cual se considera como la porción de espacio geográfico en donde está presente e interactúa de forma permanente con el ecosistema (Zunino y Zullini 2003). En términos operativos, la distribución de una especie puede ser complicada de definir por todas las variables involucradas en la interacción de una población con el ambiente. Por tal motivo, el área de distribución quedaría mejor representada si refleja el nicho ecológico efectivo de una especie en particular (Peterson et al. 2011). 
El nicho ecológico fundamental, según la definición de Hutchinson (1957), es la porción de un hiperespacio de $n$ dimensiones en la cual se desarrolla una especie. Por lo tanto, si se conocen las variables ambientales de los sitios de presencia de determinada especie, se podría dimensionar su nicho. Esta representación de nicho abarca el hábitat idóneo para la especie, es decir, el espacio ambiental traducido al área que podría ocupar potencialmente en ausencia de otras especies y con dispersión ilimitada (Hirzel et al. 2001).

El nicho fundamental de una especie puede tener regiones no ocupadas efectivamente por la especie debido a interacciones bióticas, como exclusión competitiva, depredación o falta de polinizadores, o por razones históricas, como limitaciones de dispersión o extinciones locales (Guisan y Zimmermann 2000; Austin 2002; Peterson 2006). El nicho ecológico efectivo sería, por lo tanto, aquella porción del nicho fundamental que es utilizada efectivamente por la especie (Anderson et al. 2002).

Se puede obtener información ambiental de una determinada zona del planeta y contrastarla con los intervalos viables de cada una de las dimensiones del nicho fundamental (amplitud de tolerancia de las variables ambientales), de manera que se puedan mapear aquellas zonas en las cuales estas variables coincidan con los requerimientos de la especie (Araújo y Peterson 2012). Las representaciones gráficas del área de distribución de una especie, sobrepuestas con aquellas de otras especies de un grupo taxonómico, pueden ser consideradas como indicadores de los patrones geográficos de la diversidad de una región.

Las herramientas de modelamiento predictivo de nicho ecológico se han desarrollado para inferir el área de distribución de una especie mediante la generación de mapas de presencia potencial basados en el concepto de nicho fundamental, de manera que incluyan áreas con escasos esfuerzos de colección. Estas herramientas integran datos de presencia con variables ambientales para modelar los requerimientos de hábitat de una especie y contrastar estos requerimientos con la oferta ambiental de una región. Así, se pueden modelar las áreas en donde éstas condiciones pueden ocurrir, lo cual se traduce en su distribución potencial (Guisan y Zimmermann 2000; Anderson et al. 2003; Peterson et al. 2011; Pliscoff y Fuentes-Castillo 2011).

Aunque los modelos resultantes serán limitados, tanto por la falta de equilibrio de los propios datos de presencia con las variables ambientales consideradas (Araújo y Pearson 2005), como por los sesgos de muestreo que los datos de presencia pueden presentar (Araújo y Guisan 2006), resultan en una mejor representación del área de distribución de una especie que aquella obtenida por polígonos mínimos convexos o por los propios puntos de presencia, con los cuales usualmente se representa la distribución de una especie. La distribución resultante de los modelos es potencial, a diferencia de real, porque el modelamiento predice el nicho ecológico fundamental, es decir las zonas donde las condiciones climáticas son idóneas para la especie, sin tener en cuenta factores bióticos o razones históricas que reducen el nicho ecológico fundamental a uno efectivo.

El presente trabajo utiliza información de los esfuerzos de colección de murciélagos en el Ecuador para definir áreas de distribución potencial y patrones de riqueza y diversidad basados en modelamiento predictivo de nicho. Además, discute aspectos de conservación de los resultados obtenidos en relación con las amenazas que en la 
actualidad enfrenta la vida silvestre en el Ecuador y que en mayor medida afectan al orden Chiroptera.

De acuerdo con la más reciente evaluación del estado de conservación de los murciélagos del Ecuador (Tirira 2011), se considera que 19 especies están amenazadas, esto es un $17 \%$ de la diversidad total de mamíferos del país (Tirira y Burneo 2011). Entre las principales amenazas identificadas se encuentran la deforestación y la fragmentación de bosques como consecuencia de una colonización intensiva y la expansión de la frontera agrícola (Sierra 1996; Suárez 1998; Mosandl et al. 2008; Tirira et al. 2011).

Según el mapa de vegetación remanente del Ecuador, un 37\% de la superficie del país ha perdido su cobertura de vegetación original, alteración que se concentra en su mayor parte en la región costera (Ministerio del Ambiente 2013b); de hecho, el área que presenta el mayor número de especies de murciélagos amenazados en el país es el bosque húmedo de la Costa norte, mismo que alberga un $74 \%$ del total de especies de quirópteros amenazados en el Ecuador (Tirira y Burneo 2011). Para la región oriental, de acuerdo con esta misma fuente, la pérdida de vegetación nativa alcanza un 23\%; sin embargo, en esta parte del país, apenas dos especies de murciélagos figuran como amenazadas, esto es un $10 \%$ del total de especies de quirópteros consideradas como en peligro de extinción en el Ecuador (Tirira y Burneo 2011).

Datos de presencia. Se recopilaron y validaron geográficamente datos de colección de murciélagos en distintos museos de historia natural y en la literatura. Los datos de presencia fueron tomados principalmente de dos fuentes: la colección de mastofauna del Museo de Zoología de la Pontificia Universidad Católica del Ecuador (QCAZ) y la base de datos Red Noctilio, un compendio de información asociada con colecciones de mamíferos ecuatorianos alojados en más de 75 museos de historia natural del mundo (Tirira 1995 - 2013). A estos datos se añadieron registros de presencia provenientes de zonas fronterizas del sur de Colombia y norte de Perú, obtenidos del Global Biodiversity Information Facility (GBIF 2013), que fueron revisados, validados y georreferenciados, con la finalidad de complementar vacíos de información en las zonas limítrofes de Ecuador (especialmente en la Amazonía suroriental), en las cuales no se han realizado importantes esfuerzos de colección.

Los registros duplicados y cuyas localidades resultaban dudosas o ambiguas no fueron considerados para la elaboración de los modelos predictivos, ya que los errores espaciales que pudieran contener algunos registros de las bases de datos podrían disminuir la capacidad de predicción. Aquellos datos que fueron corroborados y verificados mediante bases de datos de localidades conocidas e información de publicaciones, hasta alcanzar niveles de georreferenciación con errores inferiores a los $2 \mathrm{~km}^{2}$, fueron homogenizados mediante proyecciones de los registros con coordenadas geodésicas al Sistema Universal Transverso de Mercator (UTM). Como Datum se utilizó el World Geodetic System 1984 (WGS84).

Selección de especies. Para evitar sesgos en el análisis se escogieron aquellas especies cuyo modelo de distribución fuese confiable, tanto por tener una buena resolución taxonómica, como un alto número de registros válidos y geográficamente extendidos. 
Se generaron modelos de distribución para especies que cumplieron con los siguientes criterios: 1) están presentes en el Ecuador continental (por lo tanto, excluye las islas Galápagos); 2) han sido registradas en por lo menos seis localidades separadas entre sí por más de 10 km, con lo cual se evitaba autocorrelaciones (Dormann 2007); y 3) su taxonomía aparece resuelta; por lo tanto, no forman parte de complejos de especies documentados, para evitar posibles sesgos relacionados con la utilización de diferentes conceptos de especie en la construcción de las bases de datos (Cayuela et al. 2009) o por confusiones entre especies similares (Guisan et al. 2007).

La taxonomía seguida para las especies analizadas se basó en Tirira $(2012,2013)$. El ordenamiento taxonómico para todos los niveles es alfabético.

Variables ambientales. Se usaron 19 variables bioclimáticas derivadas de la base de datos WorldClim (Hijmans et al. 2005). Este conjunto de variables tiene mayor significación biológica que usar los promedios de temperatura y precipitación de forma independiente (Buermann et al. 2008). El algoritmo de modelamiento calcula una envoltura bioclimática para cada especie, pues busca una correlación de los datos bioclimáticos correspondientes con los datos puntuales de distribución de una especie y asume que el clima explica la mayor parte del nicho fundamental de cada especie en un marco de relativa estabilidad climática (Beaumont et al. 2007).

Algoritmo de modelamiento. Por la naturaleza de las colecciones de museos de historia natural, que mantienen datos solamente de presencia de especies en base a sus especímenes, se ha escogido el algoritmo de máxima entropía, MaxEnt (Phillips et al. 2006; Phillips y Dudik 2008), que ha tenido buenos resultados en evaluaciones comparativas (Elith et al. 2006), e incluso ha sido eficaz para predecir distribuciones de especies en comparación con modelos fisiológicos; para los cuáles, se requiere un buen conocimiento de la historia natural de la especie (Willems y Hill 2009).

MaxEnt realiza predicciones o inferencias a partir de un grupo incompleto de datos y estima la probabilidad de distribución desconocida, mediante la obtención de la distribución de máxima entropía; es decir, la más generalizada (Phillips et al. 2006).

Aunque MaxEnt puede presentar limitaciones en algunos aspectos, como en estudios de transferibilidad; en los cuales, se ha demostrado que para conjuntos de datos incompletos otros algoritmos se desempeñan de mejor forma (Lim et al. 2002; Peterson et al. 2007), se consideró que para este estudio, MaxEnt ofrecía la ventaja de ser lo suficientemente robusto para manejar conjuntos de datos de presencia, desde bastante limitados hasta amplios, en relación con la cantidad de registros de ocurrencia disponibles.

Los modelos fueron evaluados mediante el estadístico del Área Bajo la Curva ROC (Receiver Operating Characteristics), que se obtiene al graficar los valores de sensibilidad contra los valores de especificidad de acuerdo con cuántas de las presencias o ausencias observadas coinciden con las predicciones del modelo. Una vez graficada la curva ROC se calcula el área bajo dicha curva (Area Under the Curve, AUC). El AUC representa una medida simple de la precisión del modelo (Fielding y Bell 2002). La exactitud máxima de la prueba correspondería a un valor de AUC de 1 y la mínima a un valor de 0.5 (no mejor que el azar). Usualmente, valores entre 0.5-0.7 se consideran de baja precisión; 
entre 0.7 - 0.9 indican que el modelo puede tener aplicaciones útiles; mientras que un valor mayor a 0.9 indica alta precisión (Swets 1988).

Para obtener datos independientes en la matriz de confusión, los datos de presencia de cada especie fueron separados en dos grupos. El primero (grupo de entrenamiento) fue utilizado para correr el modelo; mientras que el segundo grupo (de evaluación) fue usado para verificar la capacidad predictiva del modelo resultante. De esta forma, se evitó usar el mismo conjunto de datos en la generación y evaluación del modelo, lo cual hubiera restado independencia a la evaluación (Fielding y Bell 2002). El porcentaje de datos que formaron parte de los grupos de entrenamiento y de evaluación, así como la cantidad de replicaciones que se hizo en cada ejercicio de evaluación, fueron definidos con los criterios de Pearson et al. (2007). Debido a que existe evidencia de sobrepredicción en modelos generados con MaxEnt en conjuntos de datos limitados (Papes y Gaubert 2007), se eliminaron del estudio aquellas especies que tuvieron una evaluación baja en cualquiera de las replicaciones (AUC inferior a 0.75).

Mapas de riqueza. Para cada una de las especies con un valor AUC aceptable se generó un modelo final con el uso de todos los registros disponibles. Posteriormente, se elaboró un mapa de ausencia y presencia basado en el cálculo del umbral de dos aproximaciones, proporcionadas por el propio algoritmo, que combinan la sensibilidad y la especificidad (medidas de la capacidad predictiva del modelo), lo cual ha demostrado tener resultados importantes en estudios comparativos (Liu et al. 2005), disponibles dentro de los resultados de MaxEnt.

Los mapas resultantes fueron usados para generar, mediante superposición de capas, un mapa general de riqueza de especies de murciélagos que permite visualizar patrones geográficos que pueden luego ser contrastados, por ejemplo, con zonas actualmente protegidas o aquellas que necesitan protección (Ortega-Huerta y Peterson 2004). En el presente análisis, se contrastó la riqueza potencial de murciélagos del Ecuador con el Patrimonio de Áreas Naturales del Estado (PANE; Ministerio del Ambiente 2013a), con el Mapa de Ecosistemas del Ecuador (Ministerio del Ambiente 2013b) y con los pisos climáticos del país según Tirira (2007).

El 19\% del territorio ecuatoriano se encuentra dentro del PANE, que al momento está constituido por 45 áreas protegidas que están integradas dentro del Sistema Nacional de Áreas Protegidas del Ecuador, que incluye reservas de la biósfera, biorreservas y bosques protectores (Ministerio del Ambiente 2013a). El área de protección que cubre el PANE en el Ecuador continental es del $17 \%$ del territorio total $\left(41,027 \mathrm{~km}^{2}\right.$, de una superficie total de 248,197 $\mathrm{km}^{2}$ ).

Para determinar las áreas prioritarias de investigación, como un paso inicial en esfuerzos de conservación, fue necesario entender de manera objetiva los esfuerzos de colección que se han realizado. Con el fin de obtener una superficie continua que represente el esfuerzo de colección, se interpolaron los valores correspondientes a los individuos capturados en cada localidad de colección del Ecuador mediante la técnica de Distancia Inversa Ponderada (Inverse Distance Weighted; ESRI 2012).

Las áreas prioritarias de conservación se definieron como aquellas zonas de: 1) alta riqueza de murciélagos; 2) con superficies de vegetación natural importantes; 3) zonas que actualmente no han sido consideradas dentro del PANE; 4) zonas que a 
corto o mediano plazo podrían experimentar problemas ambientales que ameriten su incorporación dentro del sistema del PANE. Para poder definir estas áreas, se empleó el mapa de riqueza potencial de murciélagos, sobre el cual se superpuso la capa de deforestación del Ministerio del Ambiente (2013b). Sobre el resultado obtenido se colocó la capa de las 45 áreas protegidas del PANE, de manera que sea posible realizar una selección visual de las zonas importantes por su riqueza, para determinar áreas que actualmente no se encuentran protegidas y aquellas que mantienen importantes extensiones de bosques nativos remanentes.

\section{Resultados}

Riqueza potencial. Se extrajeron 21,455 registros de murciélagos del Ecuador. Luego del proceso de validación de los datos se obtuvieron un total de 20,251 registros georreferenciados. Estos registros correspondieron a 162 especies de quirópteros.

Posteriormente, al eliminar los datos duplicados, de taxonomía ambigua y de aquellas especies que presentaban un número bajo de registros para su modelamiento, o aquellas cuyos valores de AUC fueron inferiores a 0.75 luego del modelamiento, los datos finales se limitaron a un total de 10,916 registros correspondientes a 81 especies (Apéndice 1).

Mediante la superposición de los 81 modelos generados se obtuvo un mapa de riqueza potencial de murciélagos (Fig. 1), mismo que representa aquellas zonas en el país que, por sus condiciones climáticas, permitirían una mayor o menor riqueza de especies de quirópteros en simpatría, en ausencia de otras variables que restrinjan sus distribuciones individuales. Los modelos finales de las especies seleccionadas se presentan en el Apéndice 2.

Las zonas más ricas en diversidad de murciélagos en el Ecuador, con un potencial de presencia superior a las 50 especies, fueron las estribaciones centro y norte orientales, entre 250 y 1,800 m de altitud, y pequeños fragmentos de bosque en la región del Chocó, en las estribaciones noroccidentales de los Andes, con una altitud promedio de 800 m; en conjunto, estas zonas sumaron una superficie total de 35,310 km² (Fig. 2).

Según el mapa de vegetación remanente del Ecuador, la zona más afectada por la deforestación es la región costera, en donde la riqueza actual de murciélagos es moderada; mientras que las zonas con mayor riqueza se concentran en la región oriental, la misma que todavía conserva importantes extensiones de bosques naturales (Fig. 3).

Áreas protegidas. Al superponer las áreas protegidas del Ecuador continental con el mapa que presentó las zonas con mayor diversidad de murciélagos (Fig. 2), solamente $1,988 \mathrm{~km}^{2}$ se encuentran protegidos, esto es un $5.6 \%$ de los $35,310 \mathrm{~km}^{2}$ de zonas con mayor riqueza. Las zonas protegidas identificadas se limitan a pequeñas áreas de los parques nacionales Yasuní, Sumaco-Napo Galeras y Sangay, la Reserva de Producción Faunística Cuyabeno y las reservas ecológicas Antisana y Cotacachi-Cayapas (Fig. 4). Por elevación, las áreas protegidas más importantes identificadas en la planicie amazónica fueron el Parque Nacional Yasuní (con una altitud de 200 a 300 m) y la Reserva de Producción Faunística Cuyabeno (entre 200 y 350 m), mientras que en las estribaciones orientales de los Andes aparecen los parques nacionales Sumaco-Napo Galeras (con un intervalo altitudinal de 400 a 3,840 m) y Sangay (entre 750 y 5,230 m, aunque la mayor parte de registros de quirópteros ocuparon altitudes inferiores a 2,000 
m). En términos absolutos, dentro de las áreas más diversas, cuatro corresponden a las estribaciones orientales de los Andes: el Parque Nacional Llanganates (62 especies de murciélagos), el Parque Nacional Sumaco - Napo Galeras (61), el Parque Nacional Sangay (60) y la Reserva Ecológica Antisana (59); mientras que en la planicie amazónica destacan el Parque Nacional Yasuní (54 especies) y la Reserva de Producción Faunística Cuyabeno (53).

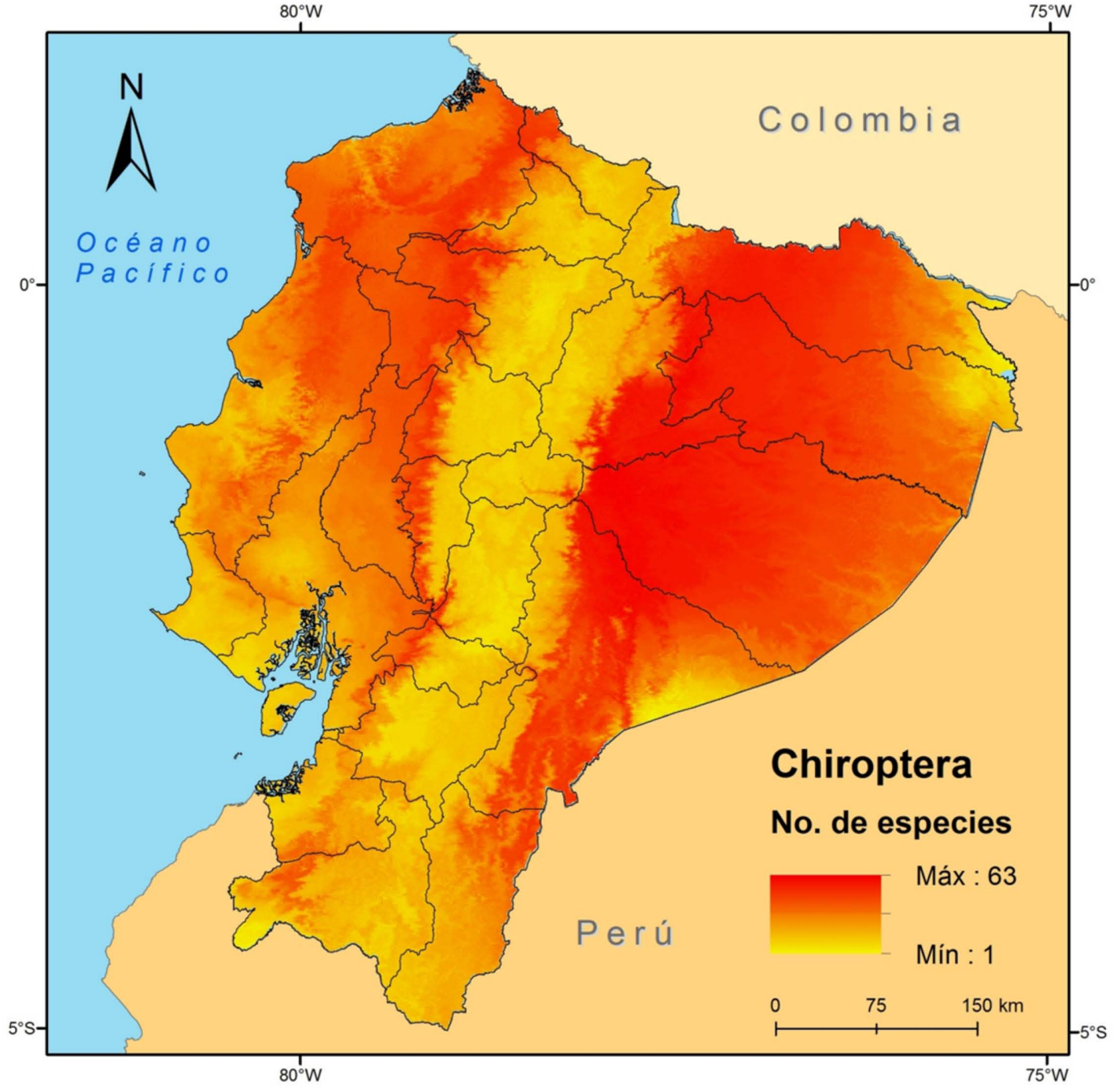

Figura 1. Riqueza potencial de murciélagos en el Ecuador, basada en la superposición de 81 modelos de distribución (Apéndice 2).

Para eliminar el efecto del tamaño del área en el análisis de la riqueza de especies, de manera que se pueda apreciar esta riqueza como una función de las variables ambientales usadas, se calculó la "riqueza relativa" como la cantidad de especies por unidad de superficie (un cálculo que a pesar de no tener un sentido biológico, ya que las especies no son entidades discretas en el espacio geográfico, permite interpretar la idoneidad del hábitat en función de las especies que albergaría tomando solo en cuenta sus características ambientales). Resulta que las áreas protegidas de mayor riqueza relativa fueron la Reserva Ecológica Cofán-Bermejo (9.6 especies por cada cien kilómetros cuadrados), la Reserva Ecológica Cayapas-Mataje (7.4), el Parque Nacional Machalilla (5.5) y la Reserva Ecológica Antisana (4.9). En comparación, áreas protegidas grandes tienen números de riqueza relativa bastante menores, como 
el Parque Nacional Yasuní (0.6 especies por cada cien kilómetros cuadrados), la Reserva de Producción Faunística Cuyabeno (0.9) y el Parque Nacional Sangay (1.2; Tabla 1).

\begin{tabular}{|c|c|c|c|c|}
\hline \multirow{8}{*}{$\begin{array}{l}\text { Tabla 1. Riqueza de } \\
\text { murciélagos según } \\
\text { las principales áreas } \\
\text { protegidas del Ecuador. }\end{array}$} & Área & Superficie (Ha) & No. de especies & Riqueza relativa $^{1}$ \\
\hline & Reserva Ecológica Cofán-Bermejo & 55,451 & 53 & 9.56 \\
\hline & Reserva Ecológica Cayapas-Mataje & 51,300 & 38 & 7.41 \\
\hline & Parque Nacional Machalilla & 56,184 & 31 & 5.52 \\
\hline & Reserva Ecológica Antisana & 120,000 & 59 & 4.92 \\
\hline & Parque Nacional Sumaco-Napo Galeras & 205,249 & 61 & 2.97 \\
\hline & Parque Nacional Llanganates & 219,707 & 62 & 2.82 \\
\hline & Parque Nacional Sangay & 517,765 & 60 & 1.16 \\
\hline \multirow{2}{*}{$\begin{array}{l}{ }^{1} \text { Número de especies } \\
\text { por cada } 100 \mathrm{~km}^{2} .\end{array}$} & Reserva de Producción Faunística Cuyabeno & 603,380 & 53 & 0.88 \\
\hline & Parque Nacional Yasuní & 982,000 & 54 & 0.55 \\
\hline
\end{tabular}

Ecosistemas. La mayor riqueza potencial obtenida coincide con cuatro grupos de ecosistemas de la Amazonía de Ecuador (según categorías de Ministerio del Ambiente 2013b; Fig. 5):

Bosques siempreverdes de tierras bajas orientales (BTBOr). Incluyen los bosques siempreverdes dentro de los sistemas de los ríos Aguarico-Putumayo-Caquetá, NapoCuraray y Tigre-Pastaza. Esto grupo de ecosistemas predijo la presencia máxima de 61 especies de murciélagos.

Bosques siempreverdes piemontanos orientales (BPOr). Incluyen los bosques siempreverdes piemontanos de las cordilleras de Galeras y del Cóndor-Kutukú y del norte y sur de la cordillera oriental de los Andes. Esto grupo de ecosistemas predijo la presencia máxima de 62 especies de murciélagos.

Bosques siempreverdes montanos bajos orientales (BMBOr). Incluyen los bosques siempreverdes montanos bajos de las cordilleras de Galeras y del Cóndor-Kutukú y del norte y sur de la cordillera oriental de los Andes. Esto grupo de ecosistemas predijo la presencia máxima de 61 especies de murciélagos.

Bosques montanos orientales (BMOr). Incluyen los bosques montanos de las cordilleras del Cóndor-Kutukú y del norte y sur de la cordillera oriental de los Andes. Esto grupo de ecosistemas predijo la presencia máxima de 56 especies de murciélagos.

En relación con la riqueza relativa, los Bosques siempreverdes de tierras bajas orientales, con una superficie de 51,556 km², presentaron 0.12 especies por cada $100 \mathrm{~km}^{2}$.

Los Bosques siempreverdes piemontanos orientales, con una superficie de 13,409 km², que equivalió a 0.46 especies por cada $100 \mathrm{~km}^{2}$. Los Bosques siempreverdes montanos bajos orientales, con 9,331 km², que equivalió a 0.65 especies por cada $100 \mathrm{~km}^{2}$. Mientras que los Bosques montanos orientales, con una superficie de 9,823 km², registraron 0.57 especies por cada $100 \mathrm{~km}^{2}$.

Áreas prioritarias. Dentro de las áreas con prioridades de investigación, para el Ecuador continental se identificaron tres zonas que merecen ser consideradas principales (Fig. 6). 
La más importante y extensa fue una sección del trópico suroriental, entre las provincias de Pastaza y Morona Santiago, principalmente dentro de la cuenca baja de los ríos Pastaza y Santiago.

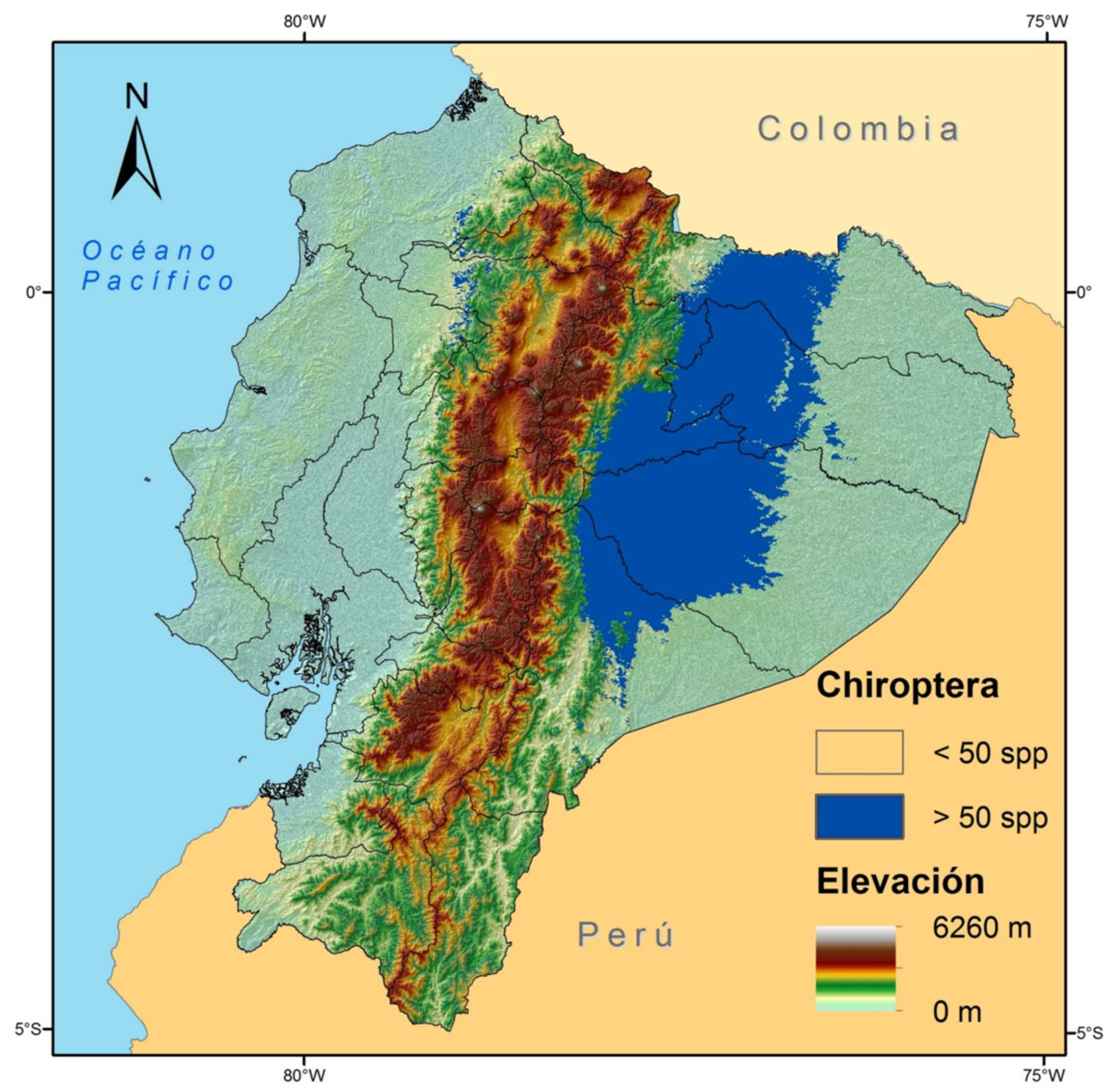

Figura 2. Zonas de mayor riqueza de murciélagos en el Ecuador, basada en la superposición de 81 modelos de distribución.

Una segunda zona importante se encuentra en los Andes del norte, en las estribaciones nororientales de la cordillera. La parte andina de esta zona mantiene áreas libres de intervención humana que permitirían considerarlas como un corredor ecológico entre el Parque Nacional Cayambe-Coca y la Reserva Ecológica Cotacachi-Cayapas.

La tercera zona en importancia involucra varios fragmentos dentro del trópico seco occidental, en las provincias de Guayas y Manabí; sin embargo, buena parte de su extensión se encuentra dentro de áreas con fuerte intervención humana que ha reducido la vegetación natural a pequeños parches remanentes. Dentro de esta zona, el único fragmento que presenta una superficie importante de remanentes de vegetación natural se encuentra en el área de bosque seco y manglares de la isla Puná y las costas continentales cercanas, en el golfo de Guayaquil (Fig. 6).

En lo referente a las áreas prioritarias para la conservación de murciélagos, la de mayor relevancia se ubica al noroccidente de la provincia de Pichincha, entre las parroquias Pacto, Mindo y San Miguel de Los Bancos (Fig. 6). Se considera que es la única extensión 
de bosque remanente suficientemente grande que podría ser conservada al occidente de la cordillera de los Andes.

La segunda área prioritaria abarca la mayor parte de la provincia de Pastaza, al sur del Parque Nacional Yasuní. La tercera área cuya conservación se considera prioritaria se ubica en las estribaciones surorientales de los Andes, entre la parte central de la provincia de Morona Santiago y el centro-norte de la provincia de Zamora Chinchipe, hasta la frontera con Perú (Fig. 6).

Figura 3. Mapa de cobertura remanente de vegetación original y áreas con la mayor diversidad de murciélagos en el Ecuador.

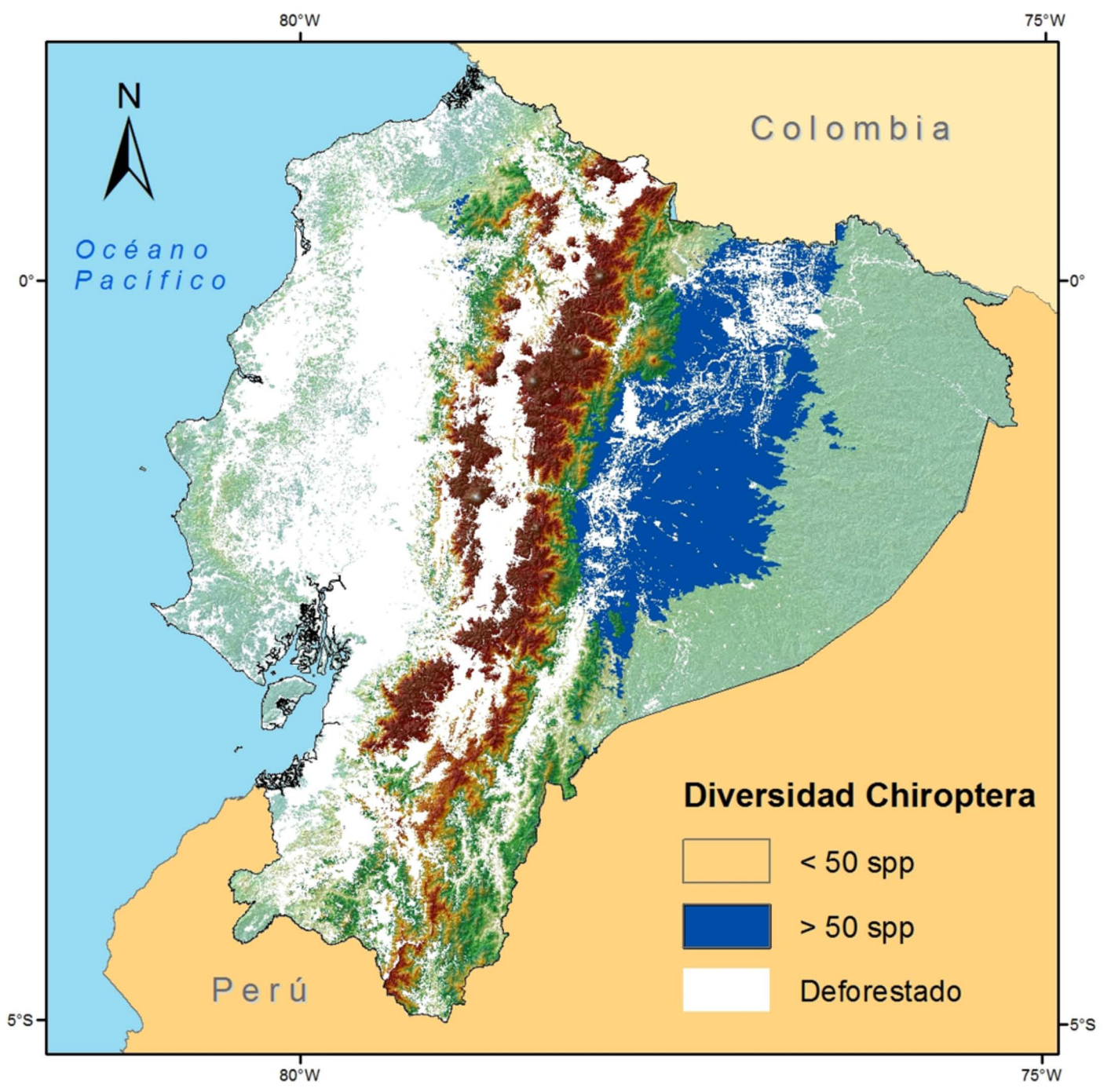

\section{Discusión}

Es necesario indicar que ciertas familias de murciélagos, como Emballonuridae, Molossidae y Vespertilionidae, estuvieron poco representadas en este estudio, debido principalmente a que el método que de forma habitual se emplea en el país para su estudio son las redes de neblina, una técnica que favorece la captura de murciélagos que vuelan en el sotobosque, como es el caso de la familia Phyllostomidae, a diferencia de las familias antes mencionadas, las cuales habitualmente vuelan sobre el dosel forestal; además, que en Ecuador, su diversidad conocida es bastante menor en relación con la familia Phyllostomidae (Tirira 2012). Pese a eso, la muestra obtenida en el presente 
estudio permitió generar modelos confiables, de manera que no sesga la discusión en términos de riqueza, distribución y aspectos de conservación, según se menciona a continuación.

El mapa que se muestra en la Fig. 1, no representa la riqueza real de murciélagos en el Ecuador, sino una tendencia de la misma. Los modelos individuales utilizados en su construcción (Apéndice 2) no han considerado criterios ecológicos, biogeográficos, ni de intervención de bosques que permitan restringir de una manera más verídica la distribución de las especies en la actualidad; por lo cual, es evidente que en estos modelos, el área de distribución real para la mayoría de las especies sería inferior al indicado en el modelo. También hay que considerar que debido a las limitaciones de muestreo, especialmente de especies de difícil registro por métodos tradicionales, como son los murciélagos insectívoros de dosel, se realizaron modelos con solo 81 de las 169 especies de quirópteros que actualmente han sido reportadas para el Ecuador (Tirira 2013); lo cual implica que existe un subestimado de la riqueza real. En todo caso, se considera que el mapa generado es importante y puede resultar útil para inferir sobre aspectos de conservación que puedan afectar la diversidad de murciélagos en el país.

Es posible que la selección de la región Amazónica como una zona que encierra las áreas de mayor riqueza de murciélagos en el Ecuador (Fig. 2), sea un artefacto explicado en parte por la falta de colecciones científicas en otras áreas del país, como por ejemplo los bosques del litoral que se perdieron debido a la agresiva expansión agrícola y ganadera desarrollada en su interior desde fines de la década de 1970 (Sierra 1996; Mosandl et al. 2008). En contraste, la mayor parte de las colecciones de murciélagos realizadas en el Ecuador se ha llevado a cabo en los últimos 20 años (Tirira 1995 - 2013).

La pérdida de cobertura de vegetación original que se observa en la región Amazónica (Fig. 3), se ubica básicamente a lo largo del sistema vial que comunica los principales centros poblados y las capitales de las provincias amazónicas, pérdida que principalmente se atribuye a procesos de colonización y deforestación intensiva relativamente recientes, ocurridos en las últimas décadas (Southgate et al. 1991; Geist y Lambin 2001), lo cual ha permitido que todavía se preserve un porcentaje importante de bosques primarios; sin embargo, las zonas en donde se registra la mayor deforestación, también coinciden con el área de mayor riqueza de murciélagos (Fig. 3), que comprenden las estribaciones orientales de los Andes, entre 250 y 1,800 m de altitud, y con un remanente de cobertura de vegetación original que alcanza un $77 \%$. La razón que explica este fenómeno, aparentemente contradictorio, es que tanto la red vial, como la frontera agrícola, han seguido patrones de colonización paralelos a las estribaciones andinas (Geist y Lambin 2001), que además son las zonas de mayor diversidad mastofaunística.

La ubicación de las áreas protegidas del Ecuador no coincide con las áreas de mayor riqueza potencial de murciélagos (Fig. 4). Pese a ser zonas de alta diversidad de mamíferos (Tirira 2007), las estribaciones bajas de los Andes, tanto orientales como occidentales, corresponden a una relativamente pequeña extensión de territorio protegido.

Las cuatro áreas protegidas que de acuerdo con el análisis realizado contienen la mayor riqueza de murciélagos se ubican en las estribaciones orientales de los Andes (Fig. 4). Todas comparten entre sí características que explican esta alta diversidad al estar ubicadas a lo largo de un gradiente altitudinal, que incluye desde tierras bajas en regiones tropicales de la planicie amazónica, hasta bosques subtropicales y templados, 
con altitudes considerables debido a la presencia de algunos volcanes en la vertiente oriental de los Andes. La ubicación geográfica y la variación altitudinal de estas áreas han prevenido el proceso de colonización, lo cual ha derivado en que su protección y conservación sea más eficiente.

Entre las zonas de mayor riqueza relativa se encuentran la Reserva Ecológica Manglares Cayapas-Mataje y el Parque Nacional Machalilla, ambas ubicadas en zonas costeras que enfrentan una alta presión humana, principalmente debido a actividades agrícolas y ganaderas, además de una alta densidad poblacional, considerablemente superior a la región amazónica (108 habitantes $/ \mathrm{km}^{2}$ en la región Costa, contra 6 habitantes $/ \mathrm{km}^{2}$ en la Amazonía; INEC 2013).

Figura 4. Áreas protegidas del Patrimonio de Âreas Naturales del Estado en relación con las zonas de mayor riqueza de murciélagos en el Ecuador. Áreas protegidas indicadas en el mapa: A. Parque Nacional Yasuní; B. PN Sumaco-Napo Galeras; C. PN Cayambe-Coca; D. PN Sangay; E. Reserva de Producción Faunística Cuyabeno. F. Reserva Ecológica Antisana; y G. RE Cotacachi-Cayapas.

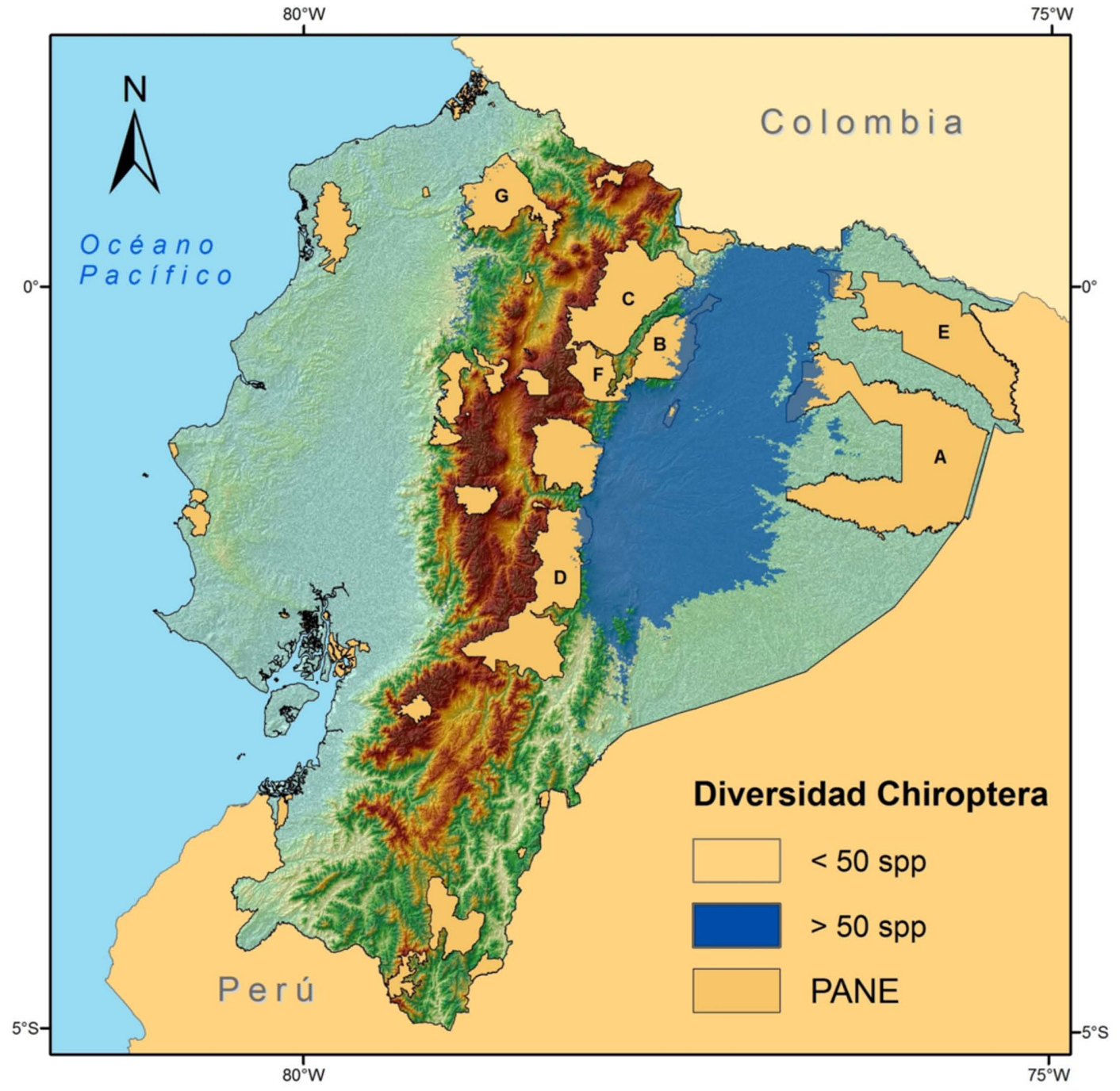

Otra área con una importante riqueza relativa es la Reserva Ecológica Cofán-Bermejo, ubicada en las estribaciones nororientales de los Andes y próxima a la frontera con Colombia, la cual presenta un amplio intervalo altitudinal (entre 400 y 2,275 m), que en cierta forma explica su alta diversidad. Una particularidad de esta reserva es que su diversidad de murciélagos no ha sido estudiada en detalle, principalmente por su cercanía con zonas ocupadas por grupos guerrilleros y paramilitares de Colombia (lo 
cual de alguna manera es también el caso de la Reserva Ecológica Manglares CayapasMataje, en el trópico noroccidental).

Es interesante que la Reserva Ecológica Antisana presente una riqueza alta de murciélagos, tanto en términos absolutos (59 especies), como relativos (4.9 especies/100 $\left.\mathrm{km}^{2}\right)$. Esta riqueza puede deberse a la superficie de la reserva $(120,000 \mathrm{ha})$ y a su amplio intervalo altitudinal (entre 1,200 y 5,760 m). Pese a esta riqueza, el Plan de Manejo oficial para la reserva reporta solamente 21 especies de murciélagos (Rivera-Rossi 2007), lo cual contrasta con registros de zonas aledañas depositados en el QCAZ, que casi triplican este número de especies. Esta relación de riqueza registrada contra riqueza potencial se repite en muchas de las áreas protegidas analizadas, debido en parte a las dificultades para obtener permisos de investigación y colección de especies dentro del PANE $y$, en parte, a lo poco rigurosas que son las investigaciones efectuadas para la elaboración de los respectivos planes de manejo.

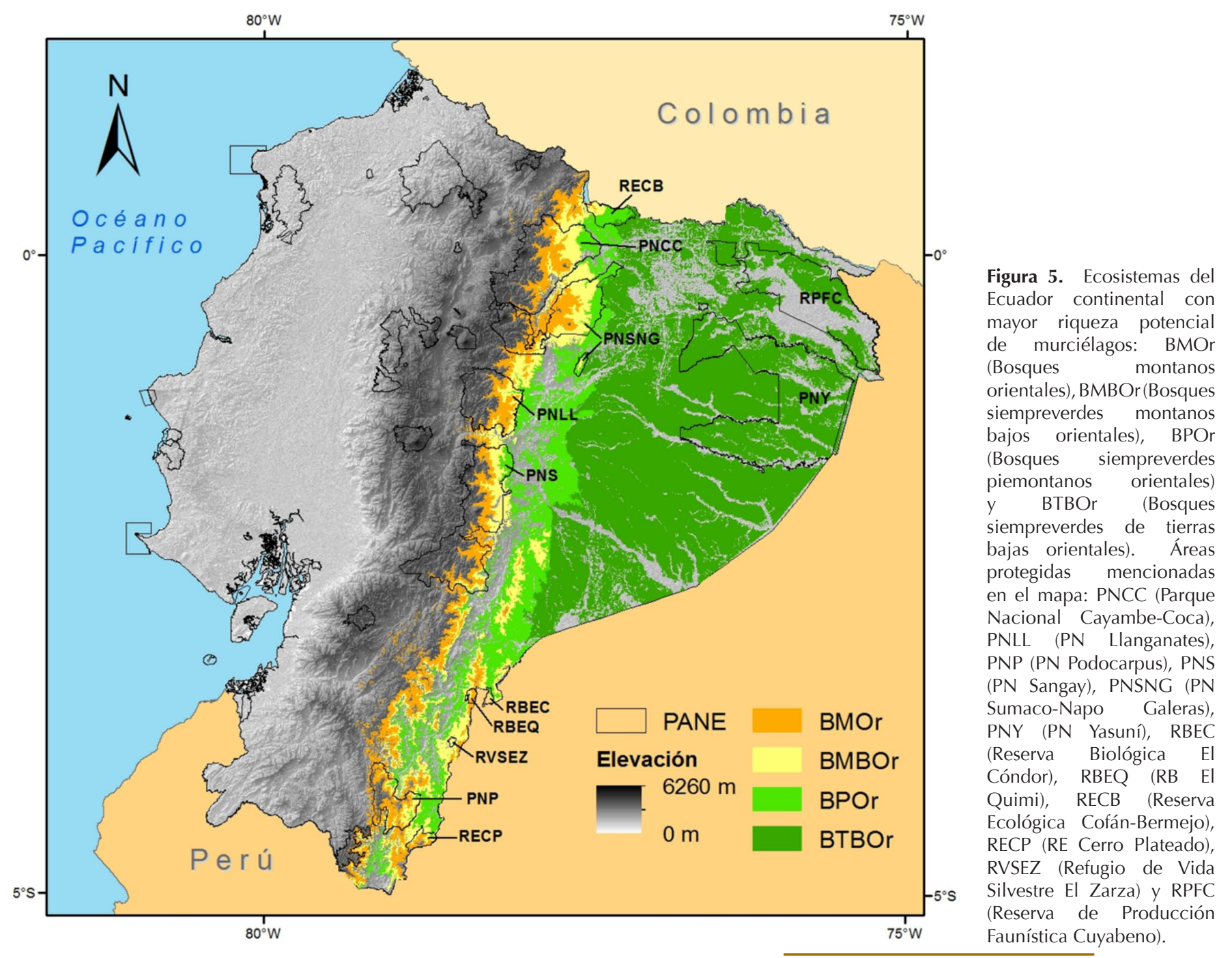

Otro ejemplo importante es el del Parque Nacional Sumaco-Napo Galeras, en el nororiente de la Amazonía ecuatoriana, entre las provincias de Napo y Orellana. Un análisis similar, en el cual se incluyeron datos de todos los mamíferos del Ecuador, presentó un resultado de 141 especies potenciales (61 de las cuales eran murciélagos), 
de 227 analizadas, que la convierten en el área más diversa del país en términos mastofaunísticos (Burneo 2010). El parque comprende dos áreas independientes, la del volcán Sumaco y la de la cordillera Galeras, con un intervalo altitudinal que va de 500 a 3,840 m (Coloma-Santos 2007). Este parque tiene además una importancia adicional en términos de conservación, pues constituye el área núcleo de la Reserva de la Biósfera Sumaco, según fue reconocida por la UNESCO (Valarezo et al. 2002).

Sobre la base de inventarios de fauna, dentro del Parque Nacional Sumaco-Napo Galeras se han documentado 81 especies de mamíferos (Coloma-Santos 2007); sin embargo, en una zona vecina al área protegida se han registrado 101 especies (Valarezo et al. 2002), lo cual unido a registros recientes del QCAZ, en la Estación Científica WildSumaco, ubicada en el área de amortiguamiento del parque, han incrementado considerablemente el listado de especies registradas en el área.

Figura 6. Áreas prioritarias para la investigación y conservación de los murciélagos en el Ecuador continental: A. Trópico suroriental; B. Andes del norte y estribaciones nororientales; C. Trópico seco occidental; D. Noroccidente de la provincia de Pichincha; E. Provincia de Pastaza; F. estribaciones surorientales (provincia de Morona Santiago y Zamora Chinchipe).

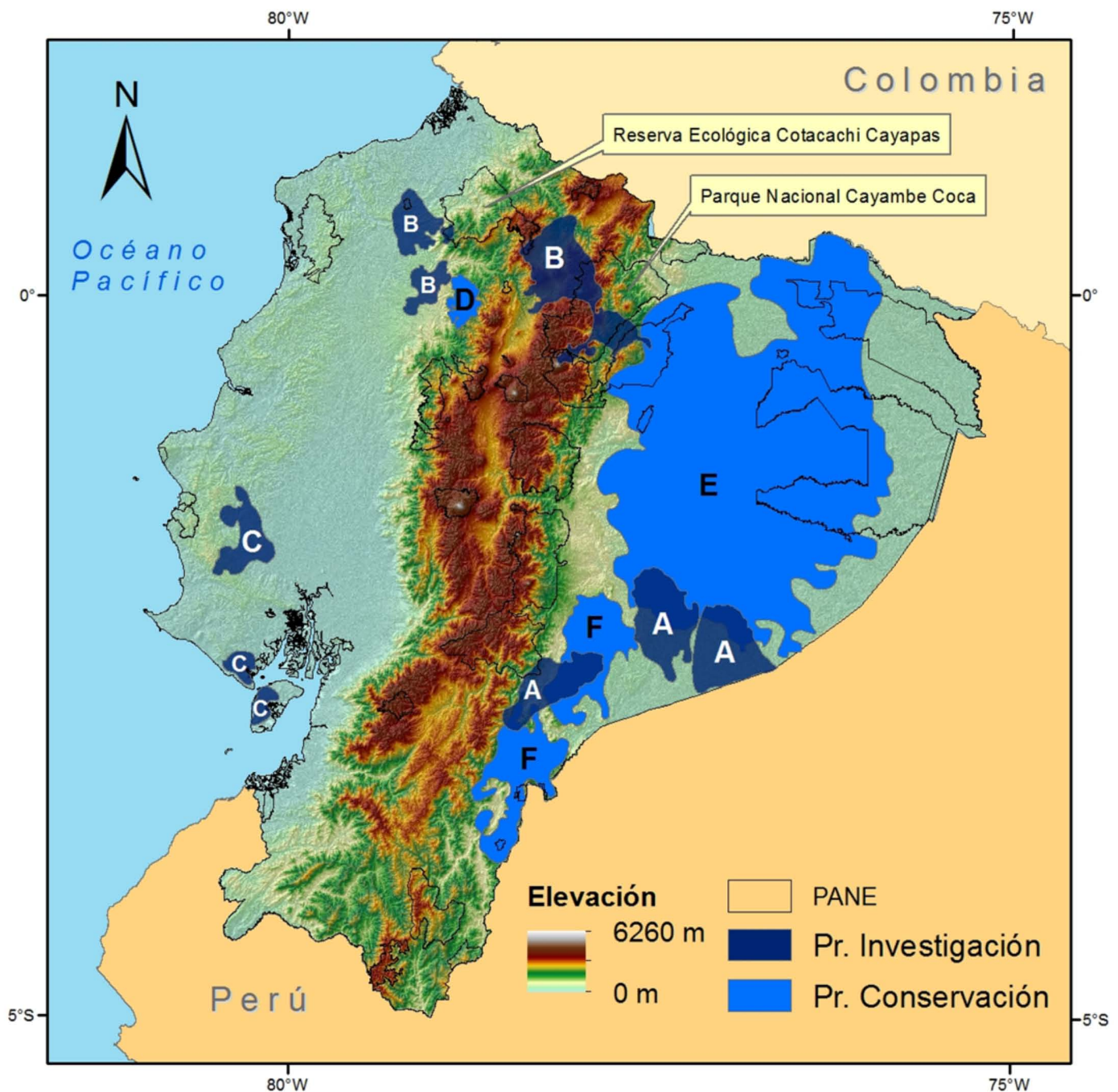

Una de las razones por las cuáles en el Parque Nacional Sumaco-Napo Galeras existe un limitado número de trabajos sistemáticos en mamíferos es el complicado relieve y el difícil acceso al área. Un trabajo en la parte alta del parque (alrededor de 2,500 m de altitud) aportó en una sola localidad 17 especies de mamíferos en tres semanas de muestreo (Lee et al. 2008). Esta situación también se refleja en la escasez de información 
para otros grupos de fauna, como las aves, para las cuales en un conteo de WildSumaco Lodge, especializado en aviturismo, reportó para esta área un total de 497 especies de aves (Nilsson 2013), mientras que en la documentación oficial se indican números de especies más bajos, de entre 280 (Coloma-Santos 2007) y 330 (Freile y Santander 2005).

La escasez de información faunística en áreas protegidas ha sido identificada por el Ministerio del Ambiente (MAE), institución gubernamental que ha planificado el desarrollo de inventarios de especies de mayor alcance y rigurosidad científica (comentario personal de Francisco Prieto, Director Nacional de Biodiversidad, MAE).

También Ilama la atención la relativa baja diversidad potencial arrojada por los modelos obtenidos para el Parque Nacional Yasuní, pues con casi un millón de hectáreas y considerado como un ícono de la conservación en el Ecuador y una de las zonas con la mayor diversidad biológica en el planeta (Rivadeneira-Roura 2007; Bass et al. 2010); tan solo reporta 54 especies de quirópteros. Estos resultados se contraponen con el criterio de Rosenzweig (1995), quien señala que zonas de mayor superficie se esperaría que alberguen un mayor número de especies, por la relación especies-área.

Los resultados del análisis de ecosistemas con mayor diversidad potencial de murciélagos respaldan las evidencias de que las estribaciones andinas representan zonas importantes para la diversidad de murciélagos (Fig. 5). Los Bosques siempreverdes montanos bajos orientales (BMBOr) son los ecosistemas que reúnen la mayor concentración de especies de murciélagos en Ecuador, con un intervalo altitudinal que va de 600 a 2,900 m y una superficie que se encuentra protegida en gran medida, ya que de los $9,331 \mathrm{~km}^{2}$ que ocupan, un $36 \%$ ha sido protegido (esto es 3,356 km²) por los parques nacionales Cayambe-Coca, Llanganates, Podocarpus, Sangay y SumacoNapo Galeras, las reservas ecológicas Antisana, Cerro Plateado y Cofán-Bermejo, las reservas biológicas El Cóndor y El Quimi y el Refugio de Vida Silvestre El Zarza.

Por otro lado, los dos grupos de ecosistemas de menor altitud en la Amazonía tienen poco territorio dentro de áreas protegidas. Los Bosques siempreverdes de tierras bajas orientales (BTBOr) tienen apenas un 22\% de su territorio protegido (esto es 11,170 de 51,556 $\mathrm{km}^{2}$ ), principalmente dentro del Parque Nacional Yasuní, la Reserva de Producción Faunística Cuyabeno y pequeñas zonas en la Reserva Ecológica CofánBermejo. Mientras que los Bosques siempreverdes piemontanos orientales (BPOr) tienen apenas un $11 \%$ protegido (1,486 de 13,409 $\left.\mathrm{km}^{2}\right)$, incluidos dentro de los parques nacionales Cayambe-Coca, Llanganates, Sangay y Sumaco-Napo Galeras y la Reserva Ecológica Cofán-Bermejo.

Dentro de las prioridades de investigación (Fig. 6), destaca el área ubicada en el trópico suroriental de Ecuador, debido a que la zona no ha sido considerada en estudios mastozoológicos previos dado su difícil accesibilidad y al conflicto limítrofe que hasta fines del siglo pasado existió con Perú, lo cual limitó la colonización humana y el acceso de investigadores para llevar a cabo colecciones de murciélagos.

En lo referente a la segunda zona con prioridad de investigación, ubicada en los Andes del norte, se ha indicado que podría servir como un corredor ecológico entre el Parque Nacional Cayambe-Coca y la Reserva Ecológica Cotacachi-Cayapas; sin embargo, las áreas contiguas hacia las estribaciones noroccidentales, dentro de las 
provincias de Esmeraldas e Imbabura, se consideran vulnerables a los problemas de deforestación relacionados con la extracción de madera y la proliferación de cultivos de palma africana y eucalipto (Buitrón 2000).

En lo referente a la tercera zona, en el trópico occidental seco, entre las provincias de Guayas y Manabí, hay numerosas iniciativas, entre ellas el ecoturismo terrestre y marino (especialmente dirigido a la observación de delfines), que se piensa promoverán la conservación de la zona, en la cual no existen áreas protegidas terrestres dentro del PANE.

En relación con las áreas prioritarias de conservación (Fig. 6), una de las razones para utilizar el mapa de riqueza acumulada de murciélagos del Ecuador, en lugar de mapas de distribución de especies clave, sombrilla, carismáticas, endémicas, únicas o en peligro de extinción, que permitan determinar las áreas prioritarias, fue la de minimizar errores de omisión (cuando erróneamente se considera ausente de una zona a una especie) y errores de comisión (cuando erróneamente se considera presente en una zona a una especie), ya que los primeros tienden a identificar áreas demasiado pequeñas y sesgadas hacia pocas especies, mientras que los segundos tienden a identificar áreas poco relevantes para la conservación (Rondinini et al. 2006).

Dentro de las áreas prioritarias seleccionadas para conservación, los bosques del noroccidente de la provincia de Pichincha tienen un aceptable estado de preservación gracias a la existencia del Bosque Protector Mindo-Nambillo, que incluye un total de 19,468 ha y se asienta en la parroquia de Mindo, una localidad en donde se desarrollan numerosas actividades de ecoturismo (especialmente observación de aves) y conservación.

El área prioritaria de conservación de la provincia de Pastaza, al sur del Parque Nacional Yasuní, se encuentra todavía en buen estado de preservación; sin embargo, prácticamente toda su superficie ha sido concesionada en bloques de exploración petrolera, de los cuáles tres se encuentran actualmente en explotación. De acuerdo con la legislación ecuatoriana vigente (Asamblea Constituyente 2008), la declaración de un área protegida no impide la explotación petrolera, o de cualquier otro tipo de recurso no renovable (siempre y cuando sea declarado como un proyecto de interés nacional por el Presidente de la República), como sucede con el Parque Nacional Yasuní y la Reserva de Producción Faunística Cuyabeno, sino que más bien conlleva mayores exigencias ambientales a las actividades de producción, lo cual podría resultar, de ser manejado bajo criterios de sostenibilidad efectivos, en mejores estrategias para la conservación de la biodiversidad.

El área prioritaria de conservación en las estribaciones surorientales del país ocupa una importante extensión de las estribaciones occidentales de la cordillera del Cóndor, en la zona limítrofe con Perú. En términos generales, esta área se encuentra bien preservada debido al conflicto limítrofe que existió entre ambos países, el cual impidió la colonización debido a la presencia de minas antipersonales. En esta zona se localizan tres áreas del PANE relativamente pequeñas: el Refugio de Vida Silvestre El Zarza y las reservas biológicas El Cóndor y El Quimi. Debido a sus particulares características, se piensa que la cordillera del Cóndor sería una buena candidata para ser declarada como Reserva de la Biósfera, que incluya la integración y ampliación de las áreas protegidas existentes, de tal manera que se salvaguarde una zona que además incluye numerosas 
concesiones mineras. Pues al igual que ocurre con el área prioritaria de la provincia de Pastaza, la región suroriental, y especialmente la cordillera del Cóndor, ha sido dividida en bloques de exploración minera; aunque todavía no existe explotación a gran escala, se piensa que debido a la agresividad de las operaciones extractivas la biodiversidad de la zona correría peligro, a diferencia de lo que ocurre con la explotación petrolera.

En la parte norte de esta misma área prioritaria de conservación se encuentra el Bosque Protector Cordilleras Kutuku y Shaimi, con una superficie de 344,000 ha, de las cuales alrededor del 95\% presenta vegetación natural. Por la extensión e importancia de esta zona, se sugiere que debería ser considerada como un área protegida dentro del sistema nacional del PANE.

Las zonas prioritarias de conservación seleccionadas no solo serían importantes para mantener un alto número de especies de murciélagos en sus áreas de influencia, sino que también servirían de conexión entre reservas ya establecidas, con lo cual se lograría un verdadero sistema de reservas integradas, que a su vez ofrecería mejores resultados de conservación a mediano y largo plazo, y además optimizaría el manejo de los limitados recursos económicos que en el Ecuador se destinan a la conservación del ambiente (Pressey et al. 1993; Margules y Pressey 2000).

Las tres áreas prioritarias de investigación y las tres áreas prioritarias de conservación identificadas serán consideradas dentro del diseño de la Estrategia para la Conservación de los Murciélagos del Ecuador, actualmente en proceso de desarrollo entre el Programa para la Conservación de los Murciélagos del Ecuador y el Ministerio del Ambiente, tanto para diseñar proyectos de investigación, como para dirigir la determinación de Áreas y Sitios Importantes para la Conservación de Murciélagos, de acuerdo con los lineamientos formulados por la Red Latinoamericana para la Conservación de Murciélagos (RELCOM 2011).

Es necesario llevar a cabo análisis teóricos y prácticos para determinar la eficacia que puedan tener los estudios de modelamientos de distribución de especies cuando están dirigidos al diseño y definición de áreas prioritarias para la conservación; en especial, si se parte del supuesto de que al tener información incompleta sobre la distribución real de una especie, el modelamiento presenta una buena aproximación, pero su eficacia deberá ser verificada a futuro (Wilson et al. 2005).

La conservación de los bosques que mantienen los patrones más altos de diversidad de murciélagos es urgente. Como impacto adicional del presente estudio se espera abrir líneas de investigación a corto plazo (como el desarrollo de inventarios y monitoreos en las áreas prioritarias de investigación determinadas) y a largo plazo, para cubrir los vacíos de información existentes.

A la Dirección de Investigaciones y Posgrados de la Pontificia Universidad Católica Agradecimientos del Ecuador, por el financiamiento del proyecto "Áreas Prioritarias de Investigación y Conservación de Mamíferos del Ecuador". A los colegas y amigos del Programa para la Conservación de los Murciélagos del Ecuador y de la Red Latinoamericana para la Conservación de los Murciélagos, por motivar estudios científicos en este importante grupo de mamíferos. A los curadores, responsables o investigadores de los museos que gentilmente proporcionaron sus catálogos de colección y alimentaron la base de datos 
Red Noctilio. A las bases de datos MaNIS y GBIF, por la información proporcionada. A

R. Owen, por invitarnos a preparar este artículo.

\section{Literatura citada}

AlbujA, L. 1982. Murciélagos del Ecuador. Primera edición. Departamento de Ciencias Biológicas, Escuela Politécnica Nacional. Quito, Ecuador.

Albuja, L. 1999. Murciélagos del Ecuador. 2a edición. Cicetrónic Cía. Ltda. Quito, Ecuador.

Albuja, L., y P. Mena-V. 2004. Quirópteros de los bosques húmedos del occidente de Ecuador. Revista Politécnica (Biología 5) 25:19-96.

Anderson, R. P., A. T. Peterson, y M. Gómez-Laverde. 2002. Using niche-based GiS modeling to test geographic predictions of competitive exclusion and competitive release in South American pocket mice. Oikos 98:3-16.

Anderson, R. P., D. Lew, y A. T. Peterson. 2003. Evaluating predictive models of species' distributions: criteria for selecting optimal models. Ecological Modelling 162:211232.

Araújo, M. B., y A. Guisan. 2006. Five (or so) challenges for species distribution modelling. Journal of Biogeography 33:1677-1688.

Araújo, M. B., y R. G. Pearson. 2005. Equilibrium of species' distributions with climate. Ecography 28:693-695.

Araújo, M. B., y A. T. Peterson. 2012. Uses and misuses of bioclimatic envelope modeling. Ecology 93:1527-1539.

Asamblea Constituyente. 2008. Constitución de la República del Ecuador. Editorial de la Asamblea Constituyente. Montecristi, Ecuador.

Austin, M. P. 2002. Spatial prediction of species distribution: an interface between ecological theory and statistical modelling. Ecological Modelling 157:101-118.

Bass, M. S., M. Finer, C. N. Jenkins, H. Kreft, D. F. Cisneros-Heredia, S. F. McCracken, N. C. A. Pitman, P. H. English, K. Swing, G. Villa, A. Di Fiore, C. C. Voigt, y T. H. Kunz. 2010. Global Conservation Significance of Ecuador's Yasuní National Park. Public Library of Science 5(1): doi: 10.1371/journal.pone.0008767.

Beaumont, L. J., A. J. Pitman, M. Poulsen, y L. Hughes. 2007. Where will species go? Incorporating new advances in climate modelling into projections of species distributions. Global Change Biology 13:1368-1385.

Buermann, W., S. Saatchi, T. B. Smith, B. R. Zutta, J. A. Chaves, B. Mila, y C. H. Graham. 2008. Predicting species distributions across the Amazonian and Andean regions using remote sensing data. Journal of Biogeography 35:1160-1176.

Buitrón, R. 2000. Documento Informativo sobre Palma Africana. El caso de Ecuador: ¿El paraíso en siete años? Boletín de Acción Ecológica 91:1-10.

Burneo, S. F. 2010. Uso de herramientas de sistemas de información geográfica y modelamiento de distribuciones para determinar zonas prioritarias de investigación y conservación de la mastofauna ecuatoriana. Disertación previa a la obtención del Título de Maestría en Biología de la Conservación. Universidad Internacional de Andalucía. Hueva, España. 
Carrera, J. P., S. Solari, P. A. Larsen, D. F. Alvarado-Serrano, A. D. Brown, C. Carrión B., J. S. Tello, y R. J. Baker. 2010. Bats of the tropical lowlands of Western Ecuador. Special Publications of the Museum of Texas Tech University 57:1-37.

Cayuela, L., D. Golicher, A. Newton, H. Kolb, F. S. de Alburquerque, E. J. M. Arets, J. R. M. Alkemade, y A. M. Pérez. 2009. Species distribution modeling in the tropics: problems, potentialities, and the role of biological data for effective species conservation. Tropical Conservation Science 2:319-352.

Coloma-Santos, A. 2007. Parque Nacional Sumaco Napo-Galeras. Pp. 219-224 in Guía del Patrimonio de Áreas Naturales Protegidas del Ecuador (ECOLAP y MAE eds.). ECOFUND, FAN, DarwinNet e IGM. Quito, Ecuador.

DormanN, C. F. 2007. Effects of incorporating spatial autocorrelation into the analysis of species distribution data. Global Ecology and Biogeography 16:129-138.

Elith, J., C. H. Graham, R. P. Anderson, M. Dudík, S. Ferrier, A. Guisan, R. J. Hijmans, F. Huettmann, J. R. Leathwick, y A. Lehmann. 2006. Novel methods improve prediction of species' distributions from occurrence data. Ecography 29:129-151.

ESRI. 2012. ArcGIS Desktop, v10.1. Environmental Systems Research Institute, Inc. Redlands, EE.UU.

Fielding, A. H., Y J. F. Bell. 2002. A review of methods for the assessment of prediction errors in conservation presence/absence models. Environmental Conservation 24:38-49.

Freile, J. F., y T. Santander. 2005. Áreas importantes para la conservación de las aves en Ecuador. Pp. 283-469 en Áreas importantes para la conservación de las aves en los Andes Tropicales: sitios prioritarios para la conservación de la biodiversidad (BirdLife International y Conservation International, eds.). Serie de Conservación de BirdLife 14. Quito, Ecuador.

Gardner, A. L. 2008 [2007]. Mammals of South America. Volumen 1: Marsupials, Xenarthrans, Shrews, and Bats (Gardner, A. L., ed.). The University of Chicago Press. Chicago, EE.UU.

GBIF. 2013. Mamíferos del Ecuador en colecciones de Perú y Colombia. Global Biodiversity Information Facility. En línea: <http://uat.gbif.org> [Fecha de acceso: 2013-08].

Geist H. J., y E. F. Lambin. 2001. What drives tropical deforestation? A meta-analysis of proximate and underlying causes of deforestation based on subnational case study evidence. LUCC Report Series 4. Ciaco Printshop. Louvain-la-Neuve, Bélgica.

Guisan, A., y N. E. Zimmermann. 2000. Predictive habitat distribution models in ecology. Ecological Modelling 135:147-186.

Guisan, A., N. E. Zimmermann, J. Elith, C. H. Graham, S. Phillips, y A. T. Peterson. 2007. What matters for predicting the occurrences of trees: techniques, data, or species' characteristics? Ecological Monographs 77:615-630.

Hijmans, R. J., S. Cameron, J. Parra, P. Jones, y A. Jarvis. 2005. Very high resolution interpolated climate surfaces for global land areas. International Journal of Climatology 25:1965-1978.

Hirzel, A. H., V. Helfer, y F. Metral. 2001. Assessing habitat-suitability models with a virtual species. Ecological Modelling 145:111-121. 
Hutchinson, G. E. 1957. Concluding remarks, Cold Springs Harbor Symposium on Quantitative Biology 415-427.

INEC. 2013. Censo de Población y Vivienda del Ecuador 2010. Instituto Ecuatoriano de Estadísticas y Censos, Quito, Ecuador. En línea: <http://www.inec.gob.ec/cpv/> [Fecha de acceso: 2013-09].

Lee, T. E., Jr., D. F. Alvarado-Serrano, R. N. Platt, y G. G. Goodwiler. 2006a. Report on a mammal survey of the Cosanga River drainage, Ecuador. Occasional Papers of the Museum of Texas Tech University 260:1-10.

Lee, T. E., Jr., J. B. Parker, y D. F. Alvarado-Serrano. 2006b. Results of a mammal survey of the Tandayapa valley, Ecuador. Occasional Papers of the Museum of Texas Tech University 250:1-9.

Lee, T. E., S. F. Burneo, M. R. Marchán, S. A. Roussos, y R. S. Vizcarra-Vásconez. 2008. The mammals of the temperate forests of Volcán Sumaco, Ecuador. Occasional Papers of the Museum of Texas Tech University 276:1-10.

Lee, T. E., JR., S. F. Burneo, T. J. Cochran, y D. Chávez. 2010. Small mammals of Santa Rosa, southwestern Imbabura Province, Ecuador. Occasional Papers of the Museum of Texas Tech University 290:1-14.

Lim, B. K., A. T. Peterson, y M. D. Engstrom. 2002. Robustness of ecological niche modeling algorithms for mammals in Guyana. Biodiversity and Conservation 11:1237-1246.

Liv, C., P. M. Berry, T. P. Dawson, y R. G. Pearson. 2005. Selecting thresholds of occurrence in the prediction of species distributions. Ecography 28:385-393.

Margules, C. R., y R. L. Pressey. 2000. Systematic conservation planning. Nature 405:243-253.

Ministerio del Ambiente. 2013a. Patrimonio de Áreas Naturales del Estado. Ministerio del Ambiente del Ecuador, Subsecretaría de Planificación Ambiental y Dirección de Información, Investigación y Educación Ambiental. Quito, Ecuador. En línea: $<$ http://web.ambiente.gob.ec/?q=node/59> [Fecha de acceso: 2013-11].

Ministerio del Ambiente. 2013b. Sistema de Clasificación de los Ecosistemas del Ecuador Continental. Proyecto Mapa de Ecosistemas del Ecuador. Ministerio del Ambiente del Ecuador, Subsecretaría de Patrimonio Natural. Quito, Ecuador.

Mosandl, R., S. Günter, B. Sтіmm, y M. Weber. 2008. Ecuador suffers the highest deforestation rate in South America. Pp. 37-40 in Gradients in a tropical mountain ecosystem of Ecuador (Beck, E., J. Bendix, I. Kottke, F. Makeschin, y R. Mosandl, eds.). Ecological Studies 198: Analysis and Synthesis. Edited Springer-Verlag. Berlín, Alemania.

Nitsson, J. 2013. Checklist of the Birds of Wildsumaco Wildlife Sanctuary and Adjacent Areas. En línea: <http://www.wildsumaco.com/html/birdssumaco.php> [Fecha de acceso: 2013-10].

Ortega-Huerta, M. A., y A. T. Peterson. 2004. Modelling spatial patterns of biodiversity for conservation prioritization in North-eastern Mexico. Diversity and Distributions 10:39-54.

PaPes, M., y P. Gaubert. 2007. Modelling ecological niches from low numbers of occurrences: assessment of the conservation status of poorly known viverrids (Mammalia, Carnivora) across two continents. Diversity and Distributions 13:890-902. 
Pearson, R. G., C. J. Raxworthy, M. Nakamura, y A. T. Peterson. 2007. Predicting species distributions from small numbers of occurrence records: a test case using cryptic geckos in Madagascar. Journal of Biogeography 34:102-117.

Peterson, A. T. 2006. Uses and requirements of ecological niche models and related distributional models. Biodiversity Informatics 3:59-72.

Peterson, A. T., M. Papes, y M. Eaton. 2007. Transferability and model evaluation in ecological niche modeling: a comparison of GARP and MaxEnt. Ecography 30:550-560.

Peterson, A. T., J. Soberón, R. G. Pearson, R. P. Anderson, E. Martínez-Meyer, M. Nakamura, y M. B. Araújo. 2011. Ecological niches and geographic distributions. Princeton University Press. Princeton, EE.UU.

Philusps, S. J., y M. Dudik. 2008. Modeling of species distributions with MaxEnt: new extensions and a comprehensive evaluation. Ecography 31:161-175.

Philuips, S. J., R. P. Anderson, y R. E. Schapire. 2006. Maximum entropy modeling of species geographic distributions. Ecological Modelling 190:231-259.

Pliscoff, P., y T. Fuentes-Castillo. 2011. Modelación de la distribución de especies y ecosistemas en el tiempo y en el espacio: una revisión de las nuevas herramientas y enfoques disponibles. Revista de Geografía Norte Grande 48:61-79.

Pressey, R. L., C. J. Humphries, C. R. Margules, R. I. Vane-Wright, y P. H. Williams. 1993. Beyond opportunism: key principles for systematic reserve selection. Trends in Ecology and Evolution 8:124-128.

RELCOM. 2011. Criterios y normativa para el establecimiento de Áreas Importantes para la Conservación de los Murciélagos (AICOMs) y Sitios Importantes para la Conservación de los Murciélagos (SICOMs). Red Latinoamericana para la Conservación de los Murciélagos. En línea: < http://www.relcomlatinoamerica. net/index.php/que-hacemos/conservacion> [Fecha de acceso: 2013-10].

Reid, F. A., M. D. Engstrom, y B. K. Lim. 2000. Noteworthy records of bats from Ecuador. Acta Chiropterologica 2:37-51.

Rivadeneira-Roura, C. 2007. Parque Nacional Yasuní. Pp. 233-242 in Guía del Patrimonio de Áreas Naturales Protegidas del Ecuador (ECOLAP y MAE, eds.). ECOFUND, FAN, DarwinNet e IGM. Quito, Ecuador.

Rivera-Rossı, J. 2007. Reserva Ecológica Antisana. Pp. 209-218 in Guía del Patrimonio de Áreas Naturales Protegidas del Ecuador (ECOLAP, y MAE, eds.). ECOFUND, FAN, DarwinNet, e IGM. Quito, Ecuador.

Rondinini, C., K. A. Wilson, L. Boitani, H. Grantham, y H. P. Possingham. 2006. Tradeoffs of different types of species occurrence data for use in systematic conservation planning. Ecology Letters 9:1136-1145.

Rosenzweig, M. L. 1995. Species diversity in space and time. Cambridge University Press. Cambridge, Reino Unido.

SierRA, R. 1996. La deforestación en el noroccidente del Ecuador 1983-1993. EcoCiencia., Quito, Ecuador.

Southgate, D., R. Sierra, y L. Brown. 1991. The causes of tropical deforestation in Ecuador: A statistical analysis. World Development 19:1145-1151. 
SuÁrez, L. 1998. La fragmentación de los bosques y la conservación de los mamíferos. Pp. 83-92 en Biología, sistemática y conservación de los mamíferos del Ecuador (Tirira, D. G., ed.). 1a. edición. Museo de Zoología, Pontificia Universidad Católica del Ecuador, Publicación Especial sobre los mamíferos del Ecuador 1. Quito, Ecuador.

Swets, J. A. 1988. Measuring the accuracy of diagnostic systems. Science 240:1285.

TiriRA, D. G. 1995-2013. Red Noctilio, Base de información no publicada sobre los mamíferos del Ecuador. Grupo Murciélago Blanco. Quito, Ecuador.

Tirira, D. G. (ed.). 1999. Mamíferos del Ecuador. Museo de Zoología, Pontificia Universidad Católica del Ecuador, y SIMBIOE, Publicación especial sobre los mamíferos del Ecuador 2. Quito, Ecuador.

Tirira, D. G. 2007. Guía de campo de los mamíferos del Ecuador. Ediciones Murciélago Blanco, Publicación especial sobre los mamíferos del Ecuador 6. Quito, Ecuador.

Tirira, D. G. 2008. Mamíferos de los bosques húmedos del noroccidente de Ecuador. Ediciones Murciélago Blanco, y Proyecto PRIMENET, Publicación especial sobre los mamíferos del Ecuador 7. Quito, Ecuador.

TiriRa, D. G. (ed.). 2011. Libro Rojo de los Mamíferos del Ecuador. 2a edición. Fundación Mamíferos y Conservación, Pontificia Universidad Católica del Ecuador, y Ministerio del Ambiente del Ecuador, Publicación especial sobre los mamíferos del Ecuador 8. Quito, Ecuador.

Tirira, D. G. 2012. Murciélagos del Ecuador: una referencia geográfica, taxonómica y bibliográfica. Pp. 235-326 en Investigación y conservación sobre murciélagos en el Ecuador (Tirira, D. G., y S. F. Burneo, eds.). Pontificia Universidad Católica del Ecuador, Fundación Mamíferos y Conservación, y Asociación Ecuatoriana de Mastozoología, Publicación especial sobre los mamíferos del Ecuador 9. Quito, Ecuador.

TiriRA, D. G. 2013. Lista actualizada de especies de mamíferos en el Ecuador. Versión 2013.2. Fundación Mamíferos y Conservación, y Editorial Murciélago Blanco. En línea: <http://www.mamiferosdelecuador.com/images/stories/portadas/Tirira2013Listamamiferos22013.pdf> [Fecha de acceso: 2013-09].

Tirira, D. G., y S. F. Burneo. 2011. Análisis, evaluación y comparaciones. Pp. 4758 en Libro Rojo de los Mamíferos del Ecuador (Tirira, D. G., ed.). 2a edición. Fundación Mamíferos y Conservación, Pontificia Universidad Católica del Ecuador, y Ministerio del Ambiente del Ecuador, Publicación especial sobre los mamíferos del Ecuador 8. Quito, Ecuador.

Tirira, D. G., F. Cuesta, y L. Suárez. 2011. Introducción. Pp. 21-28 en Libro Rojo de los Mamíferos del Ecuador (Tirira, D. G., ed.). 2a edición. Fundación Mamíferos y Conservación, Pontificia Universidad Católica del Ecuador, y Ministerio del Ambiente del Ecuador, Publicación especial sobre los mamíferos del Ecuador 8, Quito, Ecuador.

Valarezo, V., J. Gómez, L. Mejía, y Y. Célleri. 2002. Plan de Manejo de la Reserva de Biosfera Sumaco. Proyecto Gran Sumaco. Tena, Ecuador.

Willems, E. P., y R. A. Hitl. 2009. A critical assessment of two species distribution models: a case study of the Velvet Monkey (Cercopithecus aethiops). Journal of Biogeography 36:2300-2312. 
Wilson, K. A., M. I. Westphal, H. P. Possingham, y J. Elith. 2005. Sensitivity of conservation planning to different approaches to using predicted species distribution data. Biological Conservation 122:99-112.

Zunino, M., y A. Zutuns. 2003. Biogeografía. La dimensión espacial de la evolución. Fondo de la Cultura Económica. Ciudad de México, México.

Sometido: 25 de diciembre de 2013

Revisado: 28 de febrero de 2014

Aceptado: 25 de marzo de 2014

Editor asociado: Robert Owen

Diseño gráfico editorial: Gerardo Hernández

Apéndice 1

Lista de especies de murciélagos seleccionadas para el presente estudio. Se incluye el número de registros de cada especie en el Ecuador.

\begin{tabular}{rllr}
\hline No. & Familia & Especie & Registros \\
\hline 1 & Emballonuridae & Centronycteris centralis & 11 \\
2 & Emballonuridae & Cormura brevirostris & 21 \\
3 & Emballonuridae & Diclidurus albus & 10 \\
4 & Emballonuridae & Peropteryx macrotis & 36 \\
5 & Emballonuridae & Rhynchonycteris naso & 111 \\
6 & Emballonuridae & Saccopteryx bilineata & 164 \\
7 & Emballonuridae & Saccopteryx leptura & 28 \\
8 & Molossidae & Molossus currentium & 56 \\
9 & Molossidae & Molossus molossus & 539 \\
10 & Molossidae & Promops davisoni & 52 \\
11 & Mormoopidae & Mormoops megalophylla & 200 \\
12 & Noctilionidae & Noctilio albiventris & 125 \\
13 & Noctilionidae & Noctilio leporinus & 43 \\
14 & Phyllostomidae & Anoura caudifer & 227 \\
15 & Phyllostomidae & Anoura cultrata & 28 \\
16 & Phyllostomidae & Anoura fistulata & 32 \\
17 & Phyllostomidae & Anoura peruana & 450 \\
18 & Phyllostomidae & Artibeus fraterculus & 708 \\
19 & Phyllostomidae & Artibeus lituratus & 907 \\
20 & Phyllostomidae & Artibeus obscurus & 312 \\
21 & Phyllostomidae & Chiroderma salvini & 22 \\
22 & Phyllostomidae & Chiroderma trinitatum & 49 \\
23 & Phyllostomidae & Chiroderma villosum & 136 \\
24 & Phyllostomidae & Choeroniscus minor \\
25 & Phyllostomidae & Dermanura anderseni & 29 \\
& & &
\end{tabular}




\begin{tabular}{|c|c|c|c|}
\hline No. & Familia & Especie & Registros \\
\hline 26 & Phyllostomidae & Dermanura glauca & 243 \\
\hline 27 & Phyllostomidae & Dermanura gnoma & 41 \\
\hline 28 & Phyllostomidae & Dermanura rava & 270 \\
\hline 29 & Phyllostomidae & Dermanura rosenbergii & 128 \\
\hline 30 & Phyllostomidae & Diphylla ecaudata & 29 \\
\hline 31 & Phyllostomidae & Enchisthenes hartii & 103 \\
\hline 32 & Phyllostomidae & Glossophaga soricina & 786 \\
\hline 33 & Phyllostomidae & Glyphonycteris daviesi & 14 \\
\hline 34 & Phyllostomidae & Lichonycteris degener & 5 \\
\hline 35 & Phyllostomidae & Lichonycteris obscura & 11 \\
\hline 36 & Phyllostomidae & Lonchophylla concava & 44 \\
\hline 37 & Phyllostomidae & Lonchophylla robusta & 68 \\
\hline 38 & Phyllostomidae & Lonchophylla thomasi & 65 \\
\hline 39 & Phyllostomidae & Lonchorhina aurita & 96 \\
\hline 40 & Phyllostomidae & Lophostoma silvicolum & 202 \\
\hline 41 & Phyllostomidae & Mesophylla macconnelli & 106 \\
\hline 42 & Phyllostomidae & Micronycteris hirsuta & 37 \\
\hline 43 & Phyllostomidae & Micronycteris megalotis & 145 \\
\hline 44 & Phyllostomidae & Micronycteris minuta & 51 \\
\hline 45 & Phyllostomidae & Mimon crenulatum & 133 \\
\hline 46 & Phyllostomidae & Phylloderma stenops & 21 \\
\hline 47 & Phyllostomidae & Phyllostomus elongatus & 165 \\
\hline 48 & Phyllostomidae & Phyllostomus hastatus & 233 \\
\hline 49 & Phyllostomidae & Platyrrhinus albericoi & 76 \\
\hline 50 & Phyllostomidae & Platyrrhinus chocoensis & 29 \\
\hline 51 & Phyllostomidae & Platyrrhinus ismaeli & 46 \\
\hline 52 & Phyllostomidae & Platyrrhinus matapalensis & 120 \\
\hline 53 & Phyllostomidae & Platyrrhinus nigellus & 89 \\
\hline 54 & Phyllostomidae & Rhinophylla alethina & 81 \\
\hline 55 & Phyllostomidae & Rhinophylla fischerae & 96 \\
\hline 56 & Phyllostomidae & Sturnira bidens & 185 \\
\hline 57 & Phyllostomidae & Sturnira bogotensis & 33 \\
\hline 58 & Phyllostomidae & Sturnira erythromos & 303 \\
\hline 59 & Phyllostomidae & Sturnira magna & 87 \\
\hline 60 & Phyllostomidae & Sturnira oporaphilum & 181 \\
\hline 61 & Phyllostomidae & Sturnira tildae & 56 \\
\hline 62 & Phyllostomidae & Tonatia saurophila & 74 \\
\hline 63 & Phyllostomidae & Trachops cirrhosus & 83 \\
\hline 64 & Phyllostomidae & Trinycteris nicefori & 17 \\
\hline 65 & Phyllostomidae & Uroderma bilobatum & 297 \\
\hline 66 & Phyllostomidae & Vampyressa bidens & 60 \\
\hline 67 & Phyllostomidae & Vampyressa nymphaea & 55 \\
\hline 68 & Phyllostomidae & Vampyressa thyone & 242 \\
\hline 69 & Phyllostomidae & Vampyrodes caraccioli & 47 \\
\hline 70 & Phyllostomidae & Vampyrodes major & 11 \\
\hline 71 & Phyllostomidae & Vampyrum spectrum & 26 \\
\hline 72 & Thyropteridae & Thyroptera tricolor & 40 \\
\hline 73 & Vespertilionidae & Eptesicus andinus & 13 \\
\hline 74 & Vespertilionidae & Eptesicus innoxius & 46 \\
\hline 75 & Vespertilionidae & Histiotus montanus & 44 \\
\hline 76 & Vespertilionidae & Myotis albescens & 283 \\
\hline 77 & Vespertilionidae & Myotis keaysi & 85 \\
\hline 78 & Vespertilionidae & Myotis nigricans & 532 \\
\hline 79 & Vespertilionidae & Myotis oxyotus & 86 \\
\hline 80 & Vespertilionidae & Myotis riparius & 76 \\
\hline 81 & Vespertilionidae & Rhogeessa velilla & 21 \\
\hline
\end{tabular}


Modelos individuales. Emballonuridae: 1. Centronycteris centralis, 2. Cormura brevirostris, 3. Diclidurus albus, 4. Peropteryx macrotis, 5. Rhynchonycteris naso, 6. Saccopteryx bilineata, 7. S. leptura. Molossidae: 8. Molossus currentium, 9. Molossus molossus, 10. Promops davisoni. Mormoopidae: 11. Mormoops megalophylla. Thyropteridae: 12 . Thyroptera tricolor.

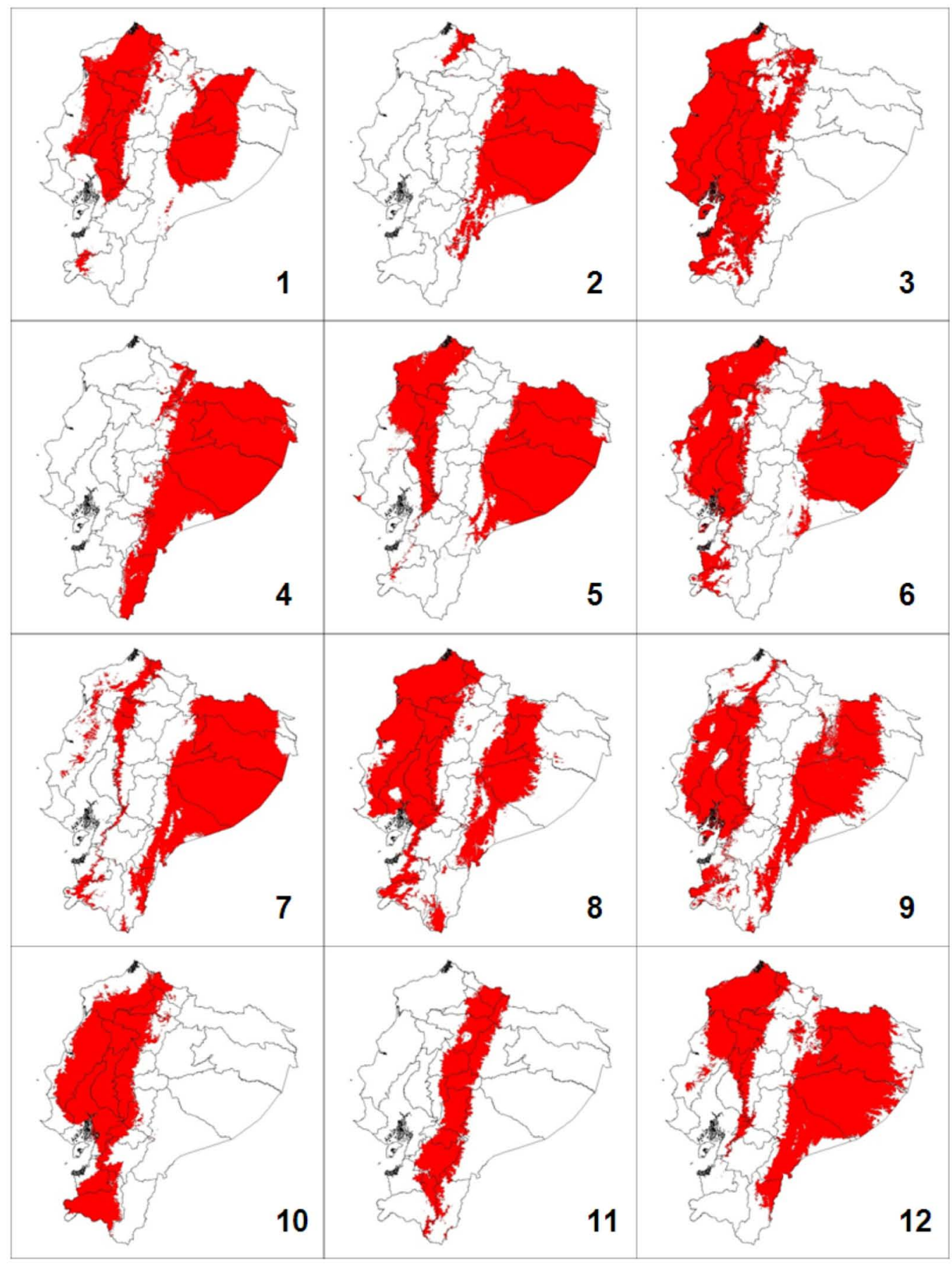




\section{Apéndice 2}

continuación Modelos individuales. Noctilionidse: 1. Noctilio albiventris, 2. N. leporinus. Phyllostomidae: 3. Anoura caudifer, 4. A. cultrata, 5. A. fistulata, 6. A. peruana, 7. Artibeus fraterculus, 8. A. lituratus, 9. A. obscurus, 10. Chiroderma salvini, 11. C. trinitatum, 12. C. villosum.

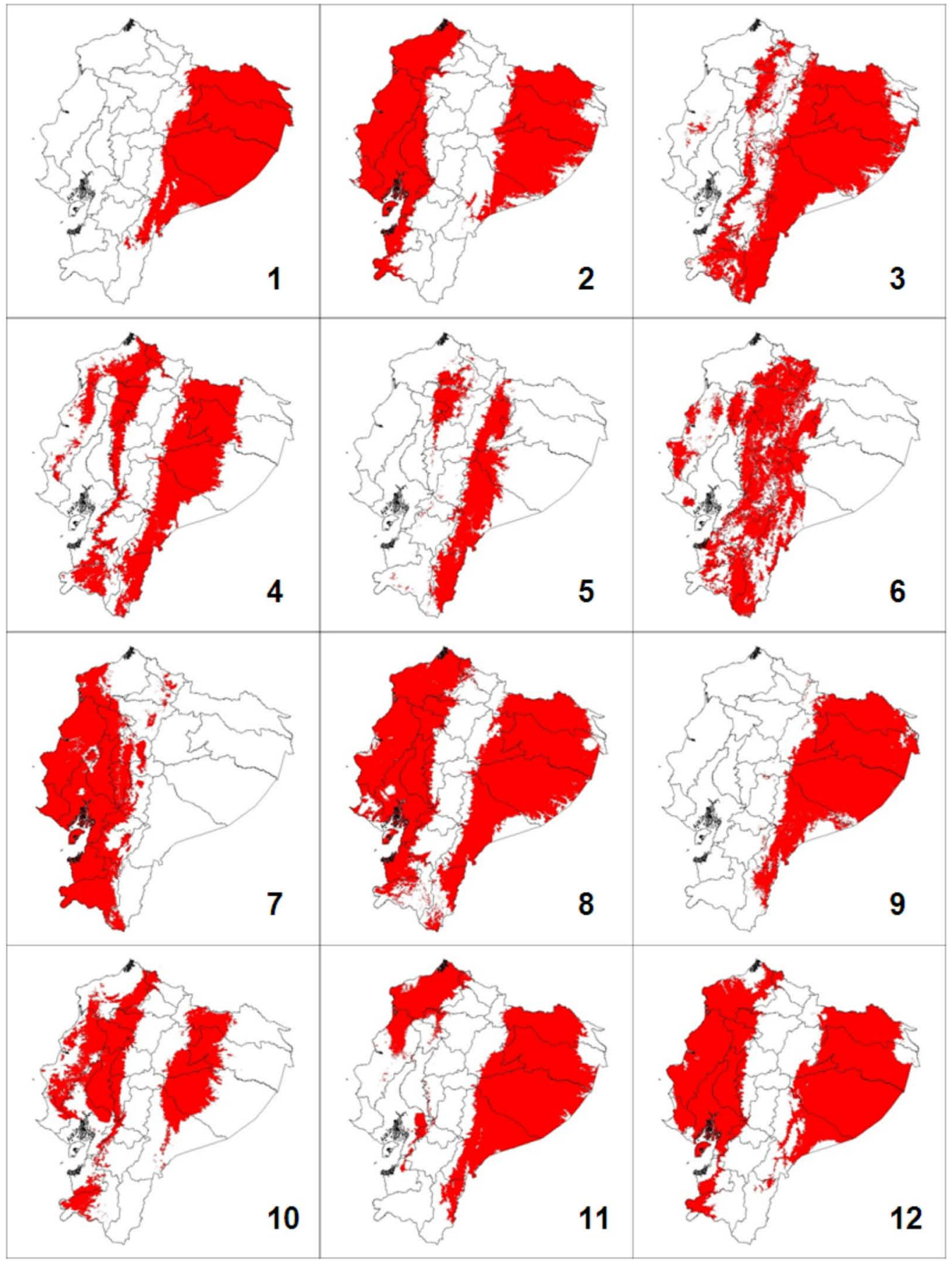


Apéndice 2

Modelos individuales. Phyllostomidae (continuación): 1. Choeroniscus minor, 2. continuación Dermanura anderseni, 3. D. glauca, 4. D. gnoma, 5. D. rava, 6. D. rosenbergii, 7. Diphylla ecaudata, 8. Enchisthenes hartii, 9. Glossophaga soricina, 10. Glyphonycteris daviesi, 11. Lichonycteris degener, 12. L. obscura.

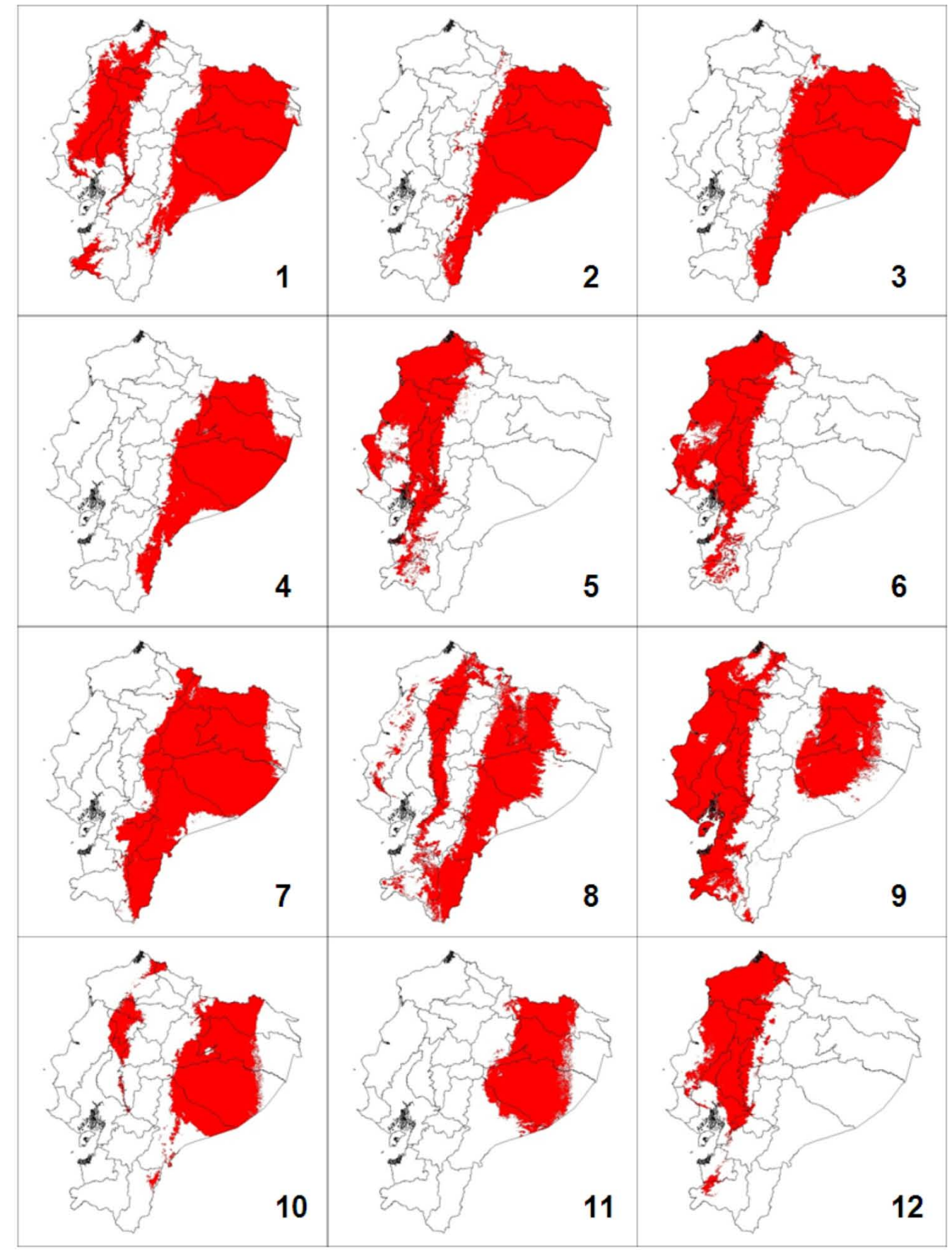




\section{Apéndice 2}

continuación Modelos individuales. Phyllostomidae (continuación): 1. Lonchophylla concava, 2. L. robusta, 3. L. thomasi, 4. Lonchorhina aurita, 5. Lophostoma silvicolum, 6. Mesophylla macconnelli, 7. Micronycteris hirsuta, 8. M. megalotis, 9. M. minuta, 10. Mimon crenulatum, 11. Phylloderma stenops, 12. Phyllostomus elongatus.

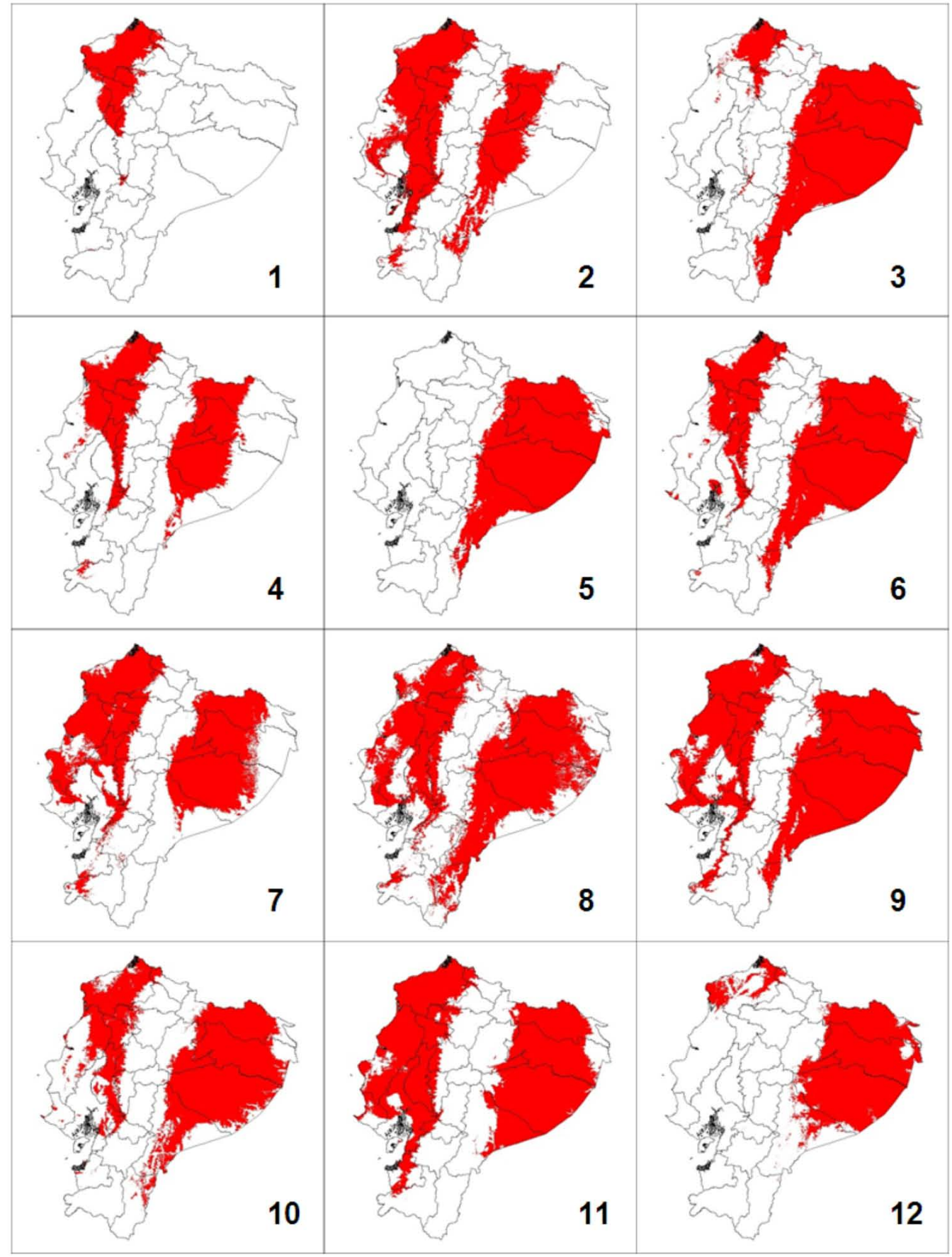


Modelos individuales. Phyllostomidae (continuación): 1. Phyllostomus hastatus, 2. continuación Platyrrhinus albericoi, 3. P. chocoensis, 4. P. ismaeli, 5. P. matapalensis, 6. P. nigellus, 7. Rhinophylla alethina, 8. R. fischerae, 9. Sturnira bidens, 10. S. bogotensis, 11. S. erythromos, 12. S. magna.

(3)




\section{Apéndice 2}

continuación Modelos individuales. Phyllostomidae (continuación): 1. Sturnira oporaphilum, 2. S. tildae, 3. Tonatia saurophila, 4. Trachops cirrhosus, 5. Trinycteris nicefori, 6. Uroderma bilobatum, 7. Vampyressa bidens, 8. V. nymphaea, 9. V. thyone, 10. Vampyrodes caraccioli, 11. V. major, 12. Vampyrum spectrum.

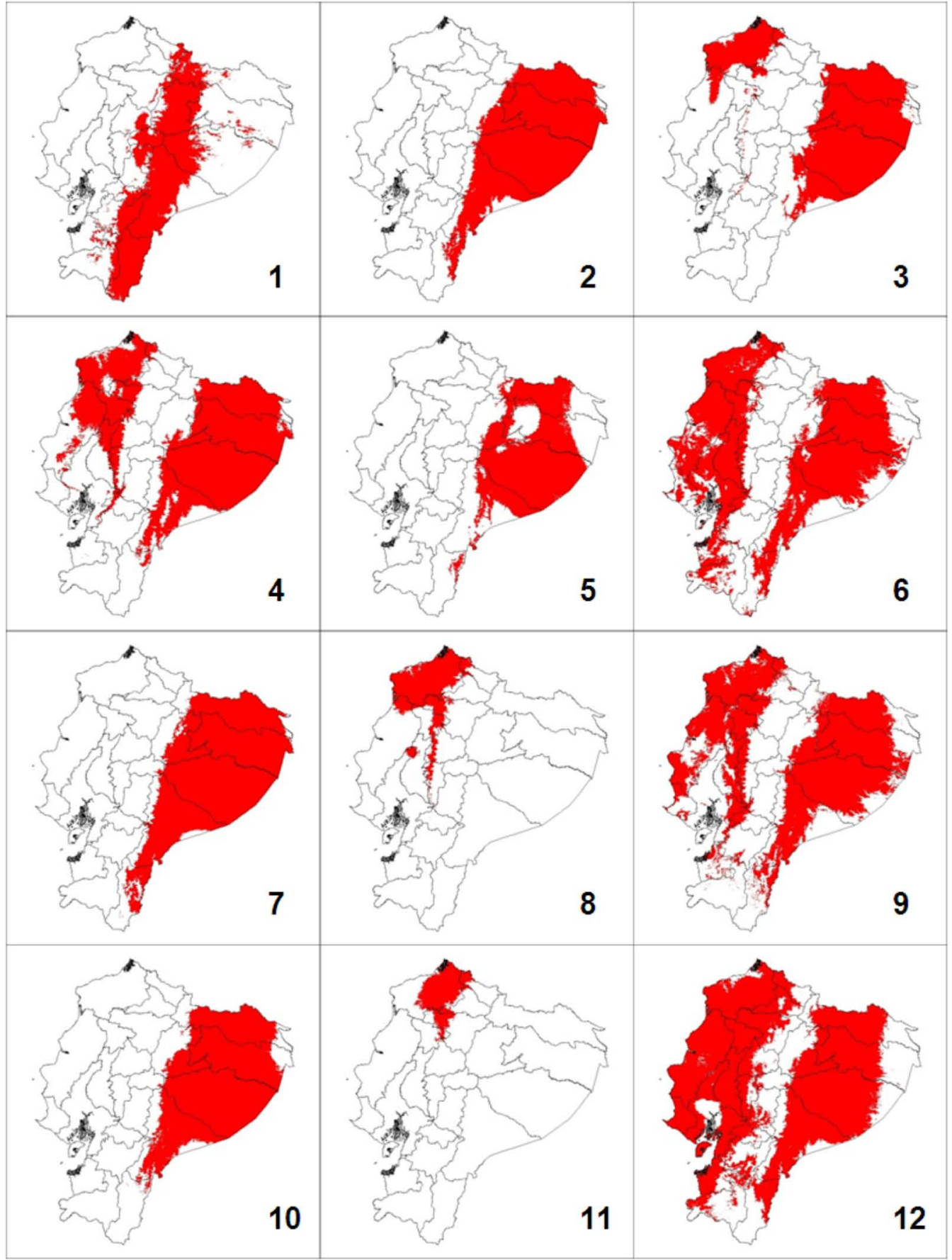


Modelos individuales. Vespertilionidae: 1. Eptesicus andinus, 2. E. innoxius, 3. Histiotus

Apéndice 2 montanus, 4. Myotis albescens, 5. M. keaysi, 6. M. nigricans, 7. M. oxyotus, 8. M. riparius, 9. Rhogeessa velilla.

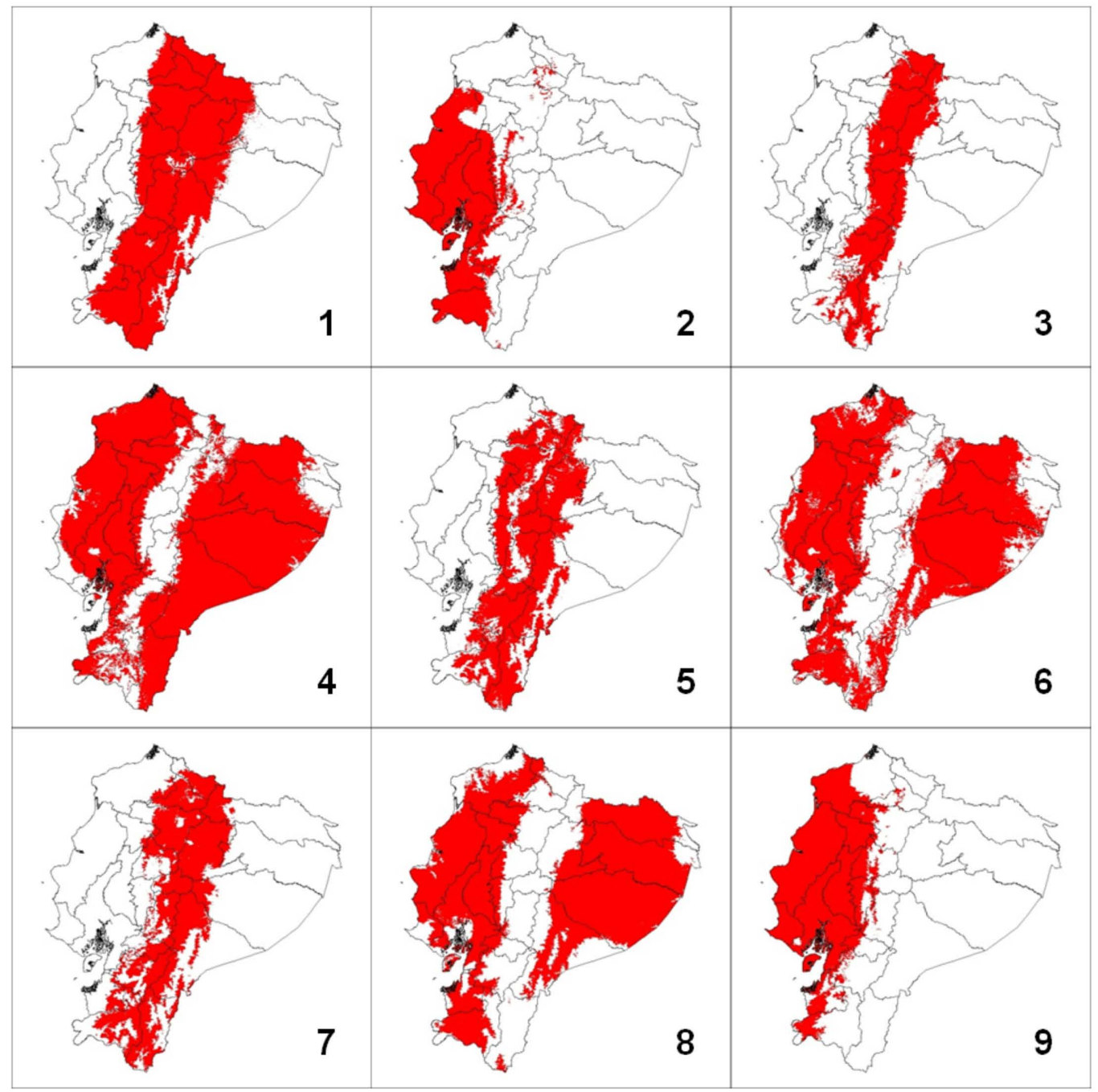

